\title{
Why do receptor-ligand bonds in cell adhesion cluster into discrete focal-adhesion sites?
}

\author{
Zhiwen Gao, ${ }^{1,2,3, *}$ and Yanfei Gao ${ }^{3,4 *}$ \\ ${ }^{1}$ Key Laboratory of Mechanics on Disaster and Environment in Western China attached to the Ministry of \\ Education of China, Lanzhou University, Lanzhou, Gansu Province, 730000, P.R. China \\ ${ }^{2}$ Department of Mechanics and Engineering Science, College of Civil Engineering and Mechanics, Lanzhou \\ University, Lanzhou, Gansu Province, 73000, P.R. China \\ ${ }^{3}$ Department of Materials Science and Engineering, University of Tennessee, Knoxville, TN 37996, USA \\ ${ }^{4}$ Materials Science and Technology Division, Oak Ridge National Laboratory, Oak Ridge, TN 37831, USA
}

\begin{abstract}
Cell adhesion often exhibits the clustering of the receptor-ligand bonds into discrete focal-adhesion sites near the contact edge, thus resembling a rosette shape or a contracting membrane anchored by a small number of peripheral forces. The ligands on the extracellular matrix are immobile, and the receptors in the cell plasma membrane consist of two types: highaffinity integrins (that bond to the substrate ligands and are immobile) and low-affinity integrins (that are mobile and not bonded to the ligands). Thus the adhesion energy density is proportional to the high-affinity integrin density. This paper provides a mechanistic explanation for the clustering/assembling of the receptor-ligand bonds from two main points: (1) the cellular contractile force leads to the density evolution of these two types of integrins, and results into a large high-affinity integrin density near the contact edge; and (2) the front of a propagating crack into a decreasing toughness field will be unstable and wavy. From this fracture mechanics perspective, the chemomechanical equilibrium is reached when a small number of patches with large receptor-ligand bond density are anticipated to form at the cell periphery, as opposed to a uniform distribution of bonds on the entire interface. Cohesive fracture simulations show that the de-adhesion force can be significantly enhanced by this nonuniform bond density field, but the de-adhesion force anisotropy due to the substrate elastic anisotropy is significantly reduced.
\end{abstract}

Keywords: receptor-ligand bond; integrin density evolution; cohesive interface model; unstable crack growth; de-adhesion anisotropy

\footnotetext{
*Correspondence information: gaozhw@1zu.edu.cn (Z. Gao) and ygao7@utk.edu (Y. Gao)
} 


\section{Introduction}

Bio-adhesive contacts have motivated a variety of biomimetic investigations mainly because the biological structures exhibit adhesion properties that are surprisingly high, low, or tunable in contrast to engineering structures. The fundamental analysis of adhesive contact has been well established in a fracture mechanics framework (Johnson, 1997). From the linear elastic fracture mechanics, the contact size is determined by balancing the energy release rate and the density of fracture energy (or adhesion energy). Nonlinear fracture analysis can also be found by the cohesive interface approach for applications in soft material adhesion or hard materials at nanoscales. The extraordinary adhesion force in fibrillar structures (such as gecko toes which has a hierarchical toe-lamellae-spatula structure with the spatula size on the order of submicrometers) is achieved mainly from the following findings: (1) the fibrillar structure can lead to a high ratio of true to nominal contact areas because of the additional compliance of these fibers; (2) the smallest scale, being the spatula in gecko adhesion, lies in the cohesive crack limit so that the de-adhesion force becomes higher than that if lying instead in the linear fracture mechanics limit; and (3) releasable adhesion can be realized either by elastic anisotropy of the substrate or due to the analogy to a peeling test that exhibits a dependence of applied energy release rate on the spatula-substrate attack angle and the peeling direction (Autumn et al., 2000; Gao et al., 2003; Yao and Gao, 2006; Gao and Bower, 2006; Chen and Gao, 2007; Ji and Gao, 2010; Zhang et al., 2013; Liu and Gao, 2015). Artificial miniature structures also design fibers with "mushroom" tip shapes so as to reduce the stress concentration at the contact edge, so that the de-adhesion force can be improved even when the adhesive contact problem falls in the linear elastic fracture mechanics limit (Spuskanyuk et al., 2008; Heepe and Gorb, 2014; Liu et al., 2015).

In contrast to the structural viewpoint in the above examples, there is a multitude of bioadhesive contacts that achieve high adhesion force by chemo-mechanical processes, with cell adhesion being the quintessential example (Freund and Lin, 2004; Deshpande et al., 2006, 2007, 2008; Lin and Freund, 2008; Maloney et al., 2008; Pathak et al., 2008, 2011; Qian et al., 2009; Wang and Gao, 2010; Gao et al., 2011; Gao, 2014; He et al., 2014; Liu et al., 2015). As shown by the fluorescent staining image in Fig. 1(a) (Huang et al., 2015), the long-chain molecular receptor-ligand bonds in the cell adhesion often cluster into a small number of focal-adhesion sites. The adhered cells resemble rosette structures or stretched membranes anchored by a small 
number of edge forces as schematically shown in Fig. 1(b). The cell interior has a low density of receptor-ligand bonds. The ligands are immobile on the extracellular matrix (ECM). Integrins in the cell membrane are binding proteins that connect to the ligands outside the cell. There are two conformational states, i.e., a low affinity (or bent) state that does not interact with ligand molecules on the ECM, and a straight state with a high affinity to the ligands. The low affinity integrins are mobile within the plasma membrane, and the high affinity ones are immobile, as schematically shown in Fig. 2.

The primary objective of this present work is to address the following questions:

(1) What factors affect the nonuniform distribution of the receptor-ligand bond density field?

(2) Why does the adhered cell take the rosette shape?

(3) How does the nonuniform receptor-ligand bond density affect the de-adhesion force?

A number of chemo-mechanical processes are involved in these problems. First, there is a conversion between the two conformational states of integrins, and the high-affinity and lowaffinity integrins should be in chemical equilibrium since this thermodynamic process is very fast (Deshpande et al., 2008). Second, as pointed out by Freund and Lin (2004) and Lin and Freund (2008), the interface behavior is controlled by the receptor-ligand bonds, but not by any kind of chemical signaling process. Therefore, the interfacial behavior in cell adhesion is primarily governed by thermodynamic and mechanical driving forces. Since only the lowaffinity integrins are mobile, a diffusional transport should be considered (Deshpande et al., 2008). Using the binder mobility of about $10 \mu \mathrm{m}^{2} \mathrm{~s}^{-1}$ and the ligand spacing of several tens of nanometers, the resulting time scale is on the order of milli-seconds. Third, the receptor-ligand bonding energy is about $1 \sim 10$ times of $k_{B} T$ with $k_{B}$ being the Boltzmann constant and $T$ being absolute temperature. Therefore, thermal fluctuations result into the stochastic behavior of the receptor-ligand molecular bonds (Qian et al., 2009; Wang and Gao, 2010). The lifetime of individual bond is on the order of 100 seconds - while the de-adhesion process can be much faster and other kinetic processes such as diffusional processes may be slower. Fourth, adhesive contact can be modulated by chemo-mechanical process away from the interface. Particularly we note that a living cell constantly reorganizes its cytoskeleton so to form or dissociate stress fibers, which apply contractile forces onto the focal adhesion contacts. In the bio-chemomechanical model developed by Deshpande et al. (2006, 2007), the processes of the formation and dissociation of stress fibers, together with the resulting cell contractility, are triggered by a 
signal (such as the concentration of $\mathrm{Ca}^{2+}$ ). The relevant characteristic time scale is on the order of minutes.

In the present work, cell adhesion is modeled as a cohesive interface fracture problem as shown in Fig. 2. Considering the vast difference in the related time scales in the above chemomechanical processes, we model the contractility by applying contractile pressures at the cell edge, neglect the stochastic behavior of receptor-ligand bonding and debonding processes, and always assume the two types of integrins in chemical equilibrium. Thus, the kinetic process in our problem is the diffusion of the low-affinity integrins. The boundary value problem consists of cell and substrate as continuous bodies and the cohesive interface as a discontinuity, where the integrin and bond densities are used as field variables. The area densities of the high-affinity and low-affinity integrins are denoted as $\xi_{H}$ and $\xi_{L}$, respectively, and the initial density of all integrins as $\xi_{0}$. The cell is modeled as an elastic circular disk (e.g., height of $2 \mu \mathrm{m}$ and radius of $20 \mu \mathrm{m})$. Clearly the cohesive interface stiffness will lead to a length scale, which will give rise to different crack-bridging characteristics when compared to the cell size, or result into the "biphasic" behavior in He et al. (2014) as will be explained in details in this work. The cohesive interface formulation enables a convenient way for finite element implementation and simulations, whereas a coupled constitutive law of mechanical response and diffusional transport should be solved.

The key idea for the formation of rosette shape in cell adhesion is given in the schematic illustration in Fig. 3. The maximum adhesion energy is reached when all receptors are highaffinity integrins, i.e., $\xi_{H} / \xi_{0} \rightarrow 1$ and $\xi_{L} / \xi_{0} \rightarrow 0$. However, due to the chemical equilibrium of high-affinity and low-affinity integrins, the initial condition usually corresponds to a very low ratio of $\xi_{H} / \xi_{L}$. Cell contraction corresponds to the application of peripheral tractions in Fig. 3 (b), and the resulting stress field will drive the conformational conversion and diffusional transport of these two types of integrins. As predicted in Deshpande et al. (2008) and will be explained in Section 3, the high-affinity integrin density field will show a radial gradient with high values lying at the contact edge. Since the adhesion energy density is proportional to $\xi_{H} / \xi_{0}$, the adhesion energy density or the interface toughness will be decreasing when moving towards the center of the contact. We now use an important conclusion from the perturbation analysis of crack front by Bower and Ortiz (1990). For a crack with straight front propagating 
into a decreasing toughness field, the crack front cannot remain straight, and will become unstable and wavy. For our problem in Fig. 3(c), in the vicinity of any contact edge point, we observe a Mode-II crack moving towards a decreasing toughness field. From Bower and Ortiz (1990), it is thus obvious that the cell shape will become rosette like as in Fig. 3(d). The final equilibrium state in Fig. 3(e) clearly balances the local energy release rate and the local toughness that depends on the integrin density of $\xi_{H} / \xi_{0}$.

To investigate the above hypothesis in Fig. 3, we first establish a potential energy functional with respect to the interface separation and integrin density fields in Section 2 . The evolution of integrin densities is studied with the focus on the role of interface stiffness and crack bridging characteristics in Section 3. In Section 4, the perturbation analysis in Bower and Ortiz (1990) is employed to determine the characteristic wavelength along the boundary for the unstable growth. When the bond density field becomes nonuniform, radially and/or angularly, a systematic computation has been performed in Section 5 to study the effects on the de-adhesion force. Conclusions will be presented in Section 6.

\section{Interface constitutive model for cell adhesion}

In the cohesive interface model, the short-range fracture process is lumped in the interface energy $\Gamma\left(\Delta_{i}\right)$, such as van der Waals interaction, or nonlinear springs such as receptorligand pairs. The interface separation is given in a vector, $\Delta_{i}$, with subscript $i$ runs from 1 to 3 . Following Rice (1992) and Gao (2010), the long-range interactions involves the calculation of energy release rate, which can be obtained from the functional derivative of the following potential energy,

$$
\Pi=\Pi_{0}+\int_{S} \Gamma\left(\Delta_{k}, \hat{\xi}_{H}\right) d S+\frac{1}{2} \int_{S} n_{i} \sigma_{i j}^{\text {self }} \Delta_{j} d S-\int_{S} n_{i} \sigma_{i j}^{\text {applied }} \Delta_{j} d S
$$

where $\hat{\xi}_{H}=\xi_{H} / \xi_{0}$ is the normalized high-affinity integrin density. Similarly, we define $\hat{\xi}_{L}=\xi_{L} / \xi_{0}$. Summation convention is implied for repeated indices. The cohesive interface model treats the interface as a two-dimensional continuum, while the deformation of the surrounding three dimensional solid leads to the first term on the right hand side, being the potential energy of the boundary value problem without any interface separation. The third term is the elastic strain energy due to interface slip with $\sigma_{i j}^{\text {self }}$ being the self-stress arising from the nonuniform interface 
separation, and the last term is the interaction energy between outside applied field, $\sigma_{i j}^{a p p l i e d}$, and $\Delta_{j}$. From Fig. 2, the applied field arises from cell contraction. The equilibrium condition is attained when the total potential energy reaches a minimum, $\delta \Pi=0$, which can be carried out from the functional calculus of the above integral.

If all the integrins are high affinity ones (i.e., $\xi_{H} / \xi_{0} \rightarrow 1$ ), we reach the ideal cohesive interface model, so that the traction force $T_{i}^{c z}$ relates to the interface separation by

$$
\begin{gathered}
T_{n}^{c z}= \begin{cases}T^{c z}(\Delta) \frac{\Delta_{n}}{\Delta}+k_{c} \zeta \dot{\Delta}_{n}, & \text { if } \Delta_{n} \geq 0 \\
k_{n} \Delta_{n}, & \text { if } \Delta_{n}<0\end{cases} \\
T_{t \alpha}^{c z}=T^{c z}(\Delta) \frac{\Delta_{t \alpha}}{\Delta}+k_{c} \zeta \dot{\Delta}_{t \alpha},
\end{gathered}
$$

where the total separation is

$$
\Delta= \begin{cases}\sqrt{\Delta_{n}^{2}+\Delta_{t 1}^{2}+\Delta_{t 2}^{2}}, & \text { if } \Delta_{n} \geq 0 \\ \sqrt{\Delta_{t 1}^{2}+\Delta_{t 2}^{2}}, & \text { if } \Delta_{n}<0\end{cases}
$$

In the above formulation, subscript $n, t_{1}$ and $t_{2}$ denote the normal direction and the two tangent directions. The distinct treatment for positive and negative $\Delta_{n}$ is to ensure impenetrable condition by setting $k_{n}>>k_{c}$. A fictitious viscosity, $\zeta$, is introduced in order to successfully simulate the snap-back instability that is intrinsic to Griffith-type cracks (Gao and Bower, 2004; Xia et al., 2007). The interface stiffness is given by $k_{c}=\xi_{0} k_{b}$, with $k_{b}$ being the receptor-ligand bond stiffness. Choosing $\xi_{0}$ in the range of $40 \sim 4000 \mu \mathrm{m}^{-2}$ and $k_{b}$ of about $0.02 \mathrm{nN} / \mu \mathrm{m}$ (Evans, 2001 ; He et al., 2014), the interface stiffness is found to be about $0.8 \sim 80 \mathrm{kPa} / \mu \mathrm{m}$.

In general, because of the integrin density evolution, the interface traction force should be

$$
T_{i}\left(\Delta_{k}, \hat{\xi}_{H}\right)=\frac{\partial \Gamma}{\partial \Delta_{i}}=\hat{\xi}_{H} T_{i}^{c z}\left(\Delta_{k}\right)
$$

The relationship, $T^{c z}(\Delta)$, in Eq. (2) can be written as a linear relationship, $T^{c z}(\Delta)=k_{c} \Delta$, as adopted in He et al. (2014), which however does not permit the fracture analysis. A bilinear model is given in the inset in Fig. 2, which has three governing parameters: the interface strength $\sigma_{\max }$, the characteristic length scale $\delta_{\max }$, and the decay length $\delta_{c}$. When implementing the 
above relationship into a nonlinear finite element framework, we need to use the material tangent, given by $J_{k l}=\partial T_{k} / \partial \Delta_{l}$ where subscripts $k$ and $l$ run as $\left(n, t_{1}, t_{2}\right)$. A tensor transform needs to be conducted for formulations in the global $x_{i}$ coordinates. Our previous works have documented the procedure to implement a cohesive interface model into the commercial finite element package, ABAQUS, via the user-defined element (UEL) subroutine (Gao and Bower, 2004; Xia et al., 2007). The challenge here is that the state variable field, $\hat{\xi}_{H}\left(x_{1}, x_{2}\right)$, evolves and is coupled to the above mechanical response of the cohesive interface.

The integrin model described below follows the work by Deshpande et al. (2008). The low-affinity and high-affinity integrins co-exist in thermodynamic equilibrium, and the low affinity ones within the plasma membrane are mobile. The chemical potential of the low affinity integrins is

$$
\mu_{L}=\mu_{L}^{0}+k_{B} T \ln \left(\frac{\xi_{L}}{\xi_{0}}\right),
$$

where $\mu_{L}^{0}$ is the reference chemical potential. The chemical potential of the high affinity integrins is, however, different, as given by

$$
\mu_{H}=\mu_{H}^{0}+k_{B} T \ln \left(\frac{\xi_{H}}{\xi_{0}}\right)+\Phi\left(\Delta_{k}\right)-F_{i}\left(\Delta_{k}\right) \Delta_{i},
$$

where $\Phi\left(\Delta_{k}\right)$ is the internal energy stored in one receptor-ligand bond and $F_{i}=\partial \Phi / \partial \Delta_{i}$ is the corresponding force. This is the bond energy, but not the elastic energy. With the increase of the elongation or separation, the bond force first increases, reaches a peak value, and then decreases to zero. Accordingly, the internal energy increases monotonically but with the change of slope with respect to $\Delta_{k}$. Referring to the cohesive interface formulation in Eq. (1), we have

$$
\begin{gathered}
\Phi\left(\Delta_{k}\right)=\frac{1}{\xi_{0}} \Gamma\left(\Delta_{k}, \hat{\xi}_{H}=1\right), \\
T_{i}=\hat{\xi}_{H} T_{i}^{c z}=\xi_{H} F_{i} .
\end{gathered}
$$

The conversion between the low and high affinity integrins is assumed to be instantaneous, so that $\mu_{H}=\mu_{L}$ is always reached everywhere and anytime. Thus the following relationship is always true, 


$$
\frac{\xi_{L}}{\xi_{H}}=\exp \left\{\frac{\mu_{H}^{0}-\mu_{L}^{0}}{k_{B} T}+\frac{1}{k_{B} T}\left[\Phi\left(\Delta_{k}\right)-F_{i}\left(\Delta_{k}\right) \Delta_{i}\right]\right\}=\beta\left(\Delta_{k}\right) .
$$

The difference in the reference chemical potential scales roughly $1 \sim 5$ times the thermal energy, i.e., $\left(\mu_{H}^{0}-\mu_{L}^{0}\right) / k_{B} T=1 \sim 5$. Thus the initial high-affinity integrin density is much lower than that of low affinity integrins. Upon stretching, the potential energy, $\Phi-F_{i} \Delta_{i}$, is negative and decreases with respect to the interface separation if $\Delta<\delta_{\max }$, so that the equilibrium condition in Eq. (8) predicts an increase in $\xi_{H}$. However, as the interface separation increases further and fracture takes place, the potential energy, $\Phi-F_{i} \Delta_{i}$, reaches a positive plateau value that is proportional to the area underneath the bilinear cohesive interface model in Fig. 2. In this stage, $\xi_{H}$ will decrease with respect to the interface separation.

The transport equation for the total integrin density, $\xi=\xi_{L}+\xi_{H}$, can be written as

$$
\frac{\partial \xi}{\partial t}=m \nabla \cdot\left(\xi_{L} \nabla \mu_{L}\right)=m k_{B} T \nabla^{2} \xi_{L}
$$

where $m$ is the mobility of the binders. Combining Eqs. (8) and (9), we have the diffusion equation for $\xi_{L}$,

$$
\frac{\partial}{\partial t}\left\{\xi_{L}\left[1+\frac{1}{\beta\left(\Delta_{i}\right)}\right]\right\}=m k_{B} T \nabla^{2} \xi_{L}
$$

with the initial condition,

$$
\left.\frac{\xi_{L}}{\xi_{H}}\right|_{0}=\exp \left\{\frac{\mu_{H}^{0}-\mu_{L}^{0}}{k_{B} T}\right\},\left.\xi_{L}\right|_{t=0}+\left.\xi_{H}\right|_{t=0}=\xi_{0}
$$

and no-flux boundary condition,

$$
\left.\left(\mathbf{n} \cdot \nabla \xi_{L}\right)\right|_{B C}=0
$$

where $\mathbf{n}$ is the surface normal at the boundary. Choosing $\xi_{0}=4444 \mu \mathrm{m}^{-2}$, the integrin spacing is thus given by $b_{l}=1 / \sqrt{\xi_{0}}=15 \mathrm{~nm}$. The binder mobility is taken as $m=10 \mathrm{mg}^{-1} \mathrm{~s}$ (Pathak et al., 2008,2011 ), so that the diffusional time scale is

$$
t^{*}=b_{l}^{2} / m k_{B} T \approx 5.3 \mathrm{~ms} .
$$

These parameters are compiled in Table 1. 
To summarize, the cohesive interface model in Eq. (1) now has a coupled chemomechanical constitutive law, with mechanical responses given in Eqs. (2) and (4) and kinetic process specified in Eqs. (8) and (10). It should be noted that the finite element framework in ABAQUS UEL subroutine does not permit a direct solution of the diffusion equation since the neighboring element information is unknown. This difficulty can be overcome by using a common block approach and the finite difference method to solve Eq. (10). Details are given in the Appendix.

\section{Result (1): The clustering of high affinity integrins}

With the interface constitutive law specified in Section 2, this Section aims to provide the mechanistic explanation of the clustering of high affinity integrins towards the contact edge. That is, we are concerned with the processes in Figs. 3(b) and 3(c). Consequently, we assume that (1) the contact problem is axisymmetric because the rosette shape will be investigated in Section 4, and (2) the evolution of high affinity integrins takes place in the cohesive interface prior to the fracture process.

The internal energy is simply given by $\Phi\left(\Delta_{i}\right)=\frac{1}{2} k_{b} \Delta^{2}$, and the potential energy is

$$
\Phi-F_{i} \Delta_{i}=-\frac{1}{2} k_{b} \Delta^{2} .
$$

The ratio of low-affinity and high-affinity integrin densities is thus

$$
\frac{\hat{\xi}_{L}}{\hat{\xi}_{H}}=\exp \left\{\frac{\mu_{H}^{0}-\mu_{L}^{0}}{k_{B} T}-\frac{k_{b} L^{2}}{2 k_{B} T}\left(\frac{\Delta}{L}\right)^{2}\right\}=\beta(\Delta),
$$

where $L$ is a characteristic length used to normalize the potential energy, thus leading to a dimensionless parameter, $\frac{k_{b} L^{2}}{2 k_{B} T}$.

One important observation of cohesive fracture analysis is that the interface stiffness naturally provides a length scale. That is, the cohesive zone length along the crack plane scales as $E_{c} / \hat{\xi}_{H} k_{c}$. If this length scale is much less than the crack size or contact size in this present problem, i.e., $E_{c} / \hat{\xi}_{H} k_{c}<<a$, we will have a small-scale-bridging (SSB) behavior, which corresponds to the linear elastic fracture mechanics (or Griffith) limit. If the cohesive zone is 
larger than the contact size, i.e., $E_{c} / \hat{\xi}_{H} k_{c}>>a$, the large-scale-bridging (LSB) behavior is obtained, in which the fracture response is governed by the interface strength. Define a dimensionless parameter for this transition,

$$
\lambda=\frac{E_{c}}{\hat{\xi}_{H} k_{c} a} .
$$

Such a crack-bridging concept has been used as a mechanistic understanding in enhancing toughness in composite structures (Bao and Suo, 1992; Shao et al., 2012). In cell mechanics, Wang and Gao (2010) noticed that a small interface stiffness will make the fracture process insensitive to the initial flaw distributions. This corresponds to the LSB limit in which the fracture is governed by interface strength so that flaws are not stress risers. He et al. (2014) modeled cell adhesion by linear springs, although the two types of integrins were not explicitly treated. They found that a large cell will lead to high stress concentration at the contact edge, while a small cell does not have this behavior, which they termed as a "biphasic" behavior. Again this is simply related to the transition from SSB to LSB with respect to the decrease of cell size. We next investigate how crack bridging characteristics affects the integrin density evolution.

Consider an axisymmetric model in Fig. 4(a), where the cell contraction is modeled by applied pressure, $\sigma_{a p p l}$, on the boundary. Modeling and geometric parameters are specified in Table 1. For the case of a perfectly bonded interface, the applied pressure of $2 \mathrm{kPa}$ (equivalent to a contraction strain of -0.1) will lead to the shear stress contour plots in Fig. 4(b). Because of the sharp geometric feature, the stress field should diverge at the notch root as shown in Fig. 5. The stress intensity factor analysis in geometries like this one can be found in Zhang et al. (2006) and Li et al. (2009). We now consider a uniform distribution of integrins, so that the interface stiffness, $\hat{\xi}_{H} k_{c}$, is constant along the entire interface. From the stress distributions in Fig. 4(c) and Fig. 5, it can be seen that the case of $\hat{\xi}_{H} k_{c}=88.88 \mathrm{kPa} / \mu \mathrm{m}$ corresponds to a small cohesive zone, about $1 \mu \mathrm{m}$ or $a / 10$ in Fig. 5 , while that of $\hat{\xi}_{H} k_{c}=0.8888 \mathrm{kPa} / \mu \mathrm{m}$ to a very large cohesive zone, about $7 \mu \mathrm{m}$ that nearly extends to the entire cell. Note the values of $1 \mu \mathrm{m}$ and $7 \mu \mathrm{m}$ for these two cases are directly read from Fig. 5 by comparing the cohesive simulation results to the 
Griffith crack solution. The estimate of the cohesive zone length by $E_{c} / \hat{\xi}_{H} k_{c}$ usually should be multiplied by a factor of $0.1 \sim 0.5$. To summarize, we note the following behavior:

- A large $\lambda$ corresponds to a cohesive zone length that is a large fraction of the contact size, i.e., the LSB behavior. In the biphasic behavior discussed by He et al. (2014), this means that no stress concentration is expected at the LSB limit, and therefore the high-affinity integrin field will not display a high gradient in the radial direction. In this case (large $\lambda$ due to small cell size, or weak bond stiffness, or stiff ECM), the cell morphology will be nearly circular.

- A small $\lambda$ corresponds to the SSB behavior. High stress concentrations will develop since the SSB response approaches the Griffith crack, and therefore a high gradient of the highaffinity integrin field will be developed. In this case (small $\lambda$ due to large cell size, or strong bond stiffness, or compliant ECM), the cell morphology will be of rosette-shape.

When the interface integrin densities evolve, we choose the initial conditions as $\left(\mu_{H}^{0}-\mu_{L}^{0}\right) / k_{B} T=4.5951$, so that $\left.\hat{\xi}_{H}\right|_{\hat{t}=0}=0.01$ and $\left.\hat{\xi}_{L}\right|_{\hat{t}=0}=0.99$. Thus at $t=0$, the interface stiffness corresponds to the weak case, $\hat{\xi}_{H} k_{c}=0.8888 \mathrm{kPa} / \mu \mathrm{m}$, which results into a large crack opening displacement in Figs. 4 and 5. The crack bridging parameter in Eq. (16) is large and the initial response is in the LSB regime. It can be seen from Eq. (15) that when there is a large relative separation, the ratio of $\xi_{L} / \xi_{H}$ will decreases, i.e., more high-affinity integrins will be clustered near large $\Delta$, leading to a high interface fracture energy density. The increase of $\hat{\xi}_{H}$ will decrease $\lambda$ in Eq. (16) and thus decrease the interface separation, and eventually a steady state will be reached. This general scenario can be validated in the test case in Fig. 6 . The rate of the applied contractile strain is a constant, $\left|\dot{\sigma}_{\text {appl }} t^{*} / E\right|=10^{-2}$, where time is normalized by the diffusion time scale $t^{*}$ in Eq. (13). The temporal evolution shows the increase of $\hat{\xi}_{H}$ and the decrease of $\hat{\xi}_{L}$ at the contact edge. We have changed from the loading rate of $\left|\dot{\sigma}_{\text {appl }} t^{*} / E\right|$ from $10^{-5}$ to 1 , but the results are only marginally different from Fig. 6 . This insensitivity has also been noticed in Deshpande et al. (2006, 2007, 2008), mainly because the time scale associated with cell contraction, being on the order of minutes, is many orders of magnitude larger than $t^{*}$ that is on the order of milli-seconds, leading to a five orders of magnitude difference. Also we note that $\hat{\xi}_{L}$ and $\beta\left(\Delta_{i}\right)$ are closely coupled in Eq. (10) even when we ignore the Laplacian term. To this 
end, an approximate solution can be obtained by setting $\xi=$ const, so as to avoid performing the time-consuming finite-difference calculations of the diffusion equation in Eq. (10). Thus, Eq. (15) leads to

$$
\hat{\xi}_{L} \approx \frac{\beta}{1+\beta}, \hat{\xi}_{H} \approx \frac{1}{1+\beta}
$$

Using this approximate solution, the shear stress distribution is found in Fig. 5 to resemble the SSB solution near the contact edge but approaches the LSB solution near the center. In other words, initially the entire interface has a low $\hat{\xi}_{H}$ and thus falls into the LSB limit, but the evolution and increase of $\hat{\xi}_{H}$ near the contact edge ensures that a SSB behavior eventually be developed at the contact edge.

\section{Result (2): A perturbation analysis for the formation of the rosette shape}

Referring back to Figs. 3(c) and 3(d), when the interface high-integrin density field decreases from the contact edge to the contact center, the subsequent contractile traction is equivalent to a crack propagating from the contact edge into a decreasing interface fracture energy field. This crack cannot maintain its straight front. Clearly this is a fully coupled problem in that the wavy crack front will change the evolution of the high-affinity integrin density field and thus the interface fracture energy. Solving this fully coupled problem is computationally challenging, and is thus out of a reasonable scope of this work. In this section, we borrow the analysis method in Bower and Ortiz (1990) to perform a perturbation analysis of the crack front, and thus to provide a mechanistic explanation of the formation of the rosette shape.

Consider a two-dimensional planar crack in $\left(x_{1}, x_{2}\right)$ plane, subjected to a stress intensity factor, $K(s)$, where $s$ is the arc length along the crack front. Now apply a small perturbation to the crack front, so that at any arbitrary location, the crack front moves by $\delta a(s)$ in the direction perpendicular to the crack front. This perturbation results into a change of the stress intensity factor, given by Rice (1985) and Gao and Rice (1986, 1987),

$$
\delta K(t) \approx \frac{1}{2 \pi} \int_{C} K(s) D(s, t) \delta a(s) d s,
$$


where $D(s, t)$ is the influence function that depends on the crack geometry. $D(s, t)$ has been derived for internal or external circular cracks by Gao $(1988,1989)$. This formulation is accurate to the first order. Bower and Ortiz (1990) developed a successive perturbation method by considering the variation in $D(s, t)$, given by

$$
\delta D(s, t) \approx \frac{1}{2 \pi} \int_{C} D(s, \varsigma) D(\varsigma, t) \delta a(\varsigma) d \varsigma .
$$

Therefore, one can gradually morph a straight or circular crack into an arbitrary crack morphology by successively and incrementally varying $a(s)$, and thus the procedure involves repeatedly evaluating the above two integrals on the crack front. The challenge lies on that the integral in Eq. (19) is undefined for general $\delta a(s)$, which can be regularized by a principal value method.

For a crack propagating into a decreasing toughness field in Fig. 3(c), the analysis is rather too complicated. Instead, we take the asymptotic view in the vicinity of any point at the contact edge, thus leading to a Mode-II crack in Fig. 7. Inspecting the distribution of high affinity integrins, we assume that the toughness decreases linearly, given by

$$
\frac{\partial K_{c}(\mathbf{x}(s))}{\partial x_{1}}=\frac{K_{c}^{0}}{\eta} .
$$

Using the formulation in Eqs. (18) and (19), the derivations in Bower and Ortiz (1990) become

$$
K_{c}+\frac{K_{c}^{0}}{\eta} \delta a(\gamma)=\left(P_{0}+\delta P\right) \hat{K}+\frac{1}{2 \pi} \int_{-\infty}^{+\infty} \frac{P_{0} \hat{K}[\delta a(s)-\delta a(\gamma)]}{(\gamma-s)^{2}} d s,
$$

where the influence function takes the form of $1 /(\gamma-s)^{2}$, and $P_{0}$ is the applied load such that $P_{0} \hat{K}=K_{c}^{0}$. Now perturb the straight crack into

$$
\delta a\left(x_{2}\right)=A_{0}-A_{1} \cos \left(\frac{2 \pi x_{2}}{y}\right)
$$

and substitute it into Eq. (21). Thus the first order perturbation solution gives a critical wavelength,

$$
y_{c r t}=\pi \eta \text {. }
$$

The above result suggests that once the integrin density has a biased distribution as in Fig. 6, the crack front will become unstable with a wavelength that is comparable to, or a few 
times, the characteristic length over which the high-affinity integrin density varies. As already been explained in Fig. 5, a small $\lambda$ corresponds to the SSB behavior, which leads to a steep gradient of $\hat{\xi}_{H}$ and thus a small $\eta / a$. In this case (small $\lambda$ due to large cell size, or strong bond stiffness, or compliant ECM), $y_{c r t}$ is small when compared to the cell perimeter, so that the cell morphology will be of rosette-shape. In contrast, the LSB behavior (large $\lambda$ due to small cell size, or weak bond stiffness, or stiff ECM) leads to a slow variation of $\hat{\xi}_{H}$ and thus a very large $\eta / a$. Therefore, no perturbation will be developed along the cell periphery. This is the mechanism behind the biphasic behavior in He et al. (2014).

While the above perturbation analysis interprets the schematic in Fig. 3(d), it is unable to obtain the final equilibrium morphology in Fig. 3(e) since a fully coupled problem has to be solved. Although this will be left for a future work, Fig. 7 provides a schematic illustration of the successive perturbation result by Bower and Ortiz (1990) that helps visualize the subsequent evolution. Their results show that the straight crack is initially added with a sinusoidal perturbation, which gradually grows and forms a protrusion-like crack morphology. It is thus anticipated the evolution from Fig. 3(d) to Fig. 3(e) will demonstrate a similar trend, i.e., a number of rosette lobes will developed.

\section{Result (3): Effects of distributed bond densities on the de-adhesion force}

In the Introduction, we have discussed the structural design of bio-adhesive contacts that lead to significantly enhanced adhesion force. One example along this line is the releasable adhesion by using the elastic anisotropy. Chen and Gao (2007) has derived analytical solutions in two-dimensional contact and found that if the pulling force is applied in an elastically compliant direction, the de-adhesion force will maximize. In a three-dimensional simulation, Liu and Gao (2015) confirmed the above solution but also found the degree of de-adhesion force anisotropy is much lower than the 2D result in Chen and Gao (2007), and this anisotropy vanishes as the contact response transitions from SSB to LSB behavior. In these studies, the interface adhesion energy density is considered to be uniform. In this section, the role of the clustering of highaffinity integrins near the contact edge is investigated on the de-adhesion force. Following the above studies, the ECM is taken as transversely isotropic, with the elastic constants given in 
Table 2. In the notation used by Chen and Gao (2007), the anisotropy is measured by the following parameter:

$$
\frac{L_{22}}{L_{11}}=\frac{\alpha_{2}}{\alpha_{1}} \sqrt{\frac{E_{t}}{E_{p}}}, \alpha_{1}=\frac{1}{\sqrt{1-v_{p}^{2}}}, \alpha_{2}=\frac{1}{\sqrt{1-v_{t p}^{2} E_{p} / E_{t}}},
$$

where subscript $t$ and $p$ denote the out-of-plane and in-plane directions (Bower, 2009). The second set of elastic constants in Table 2 corresponds to a fictitious material with an extreme value of $L_{22} / L_{11}$, in order to ensure a large anisotropy in the de-adhesion force with respect to the pulling direction.

Simulations that couple mechanical response and diffusional transport are extremely time consuming. Without the loss of generality, we adopt the bilinear cohesive interface model in Fig. 2 and assume a given and fixed distribution of the high-affinity integrins. In the reference cohesive interface model, there are two tuning parameters, $\delta_{\max }$ and $\hat{\xi}_{H}^{0} \sigma_{\max }$ where $\hat{\xi}_{H}^{0}$ is the high-affinity integrin density in the uniform distribution. We keep their products constant, but change the ratio of $\hat{\xi}_{H}^{0} \sigma_{\max } / \delta_{\max }$ so as to obtain a wide range of crack bridging behavior. For a radial distribution, following the suggestion in He et al. (2014), we use

$$
\frac{\hat{\xi}_{H}(r)}{\hat{\xi}_{H}^{0}}=f_{N}(r)=\frac{N+2}{2}\left(\frac{r}{a}\right)^{N},
$$

where $N=0,1,2 \ldots$, and an example is given in Fig. $8($ a). For both radial and angular distributions that mimic the bond density field in Fig. 1, we adopt

$$
\frac{\hat{\xi}_{H}(r)}{\hat{\xi}_{H}^{0}}=f_{N M}(r)=\frac{N+2}{2}\left(\frac{r}{a}\right)^{N}[1+\cos (M \theta)],
$$

where $M=1,2 \ldots$, and an example of $N=4$ and $M=6$ is presented in Fig. 8(b).

The test example in Fig. 9 corresponds to a crack bridging behavior close to the SSB limit because of the large ratio of $\hat{\xi}_{H}^{0} \sigma_{\max } / \delta_{\max }$, so that the pulling force versus displacement curves show sudden load drops that correspond to the crack nucleation. Results in Fig. 9(b) compare three types of bond distributions, including the uniform one with $\hat{\xi}_{H}^{0}$ and the biased ones in Eqs. (25) and (26), and their crack opening displacement (COD) contours at the onset of crack nucleation are given in Figs. 10(a), 10(c) and 10(d), respectively. In these plots, the crack front is delineated by the COD contours in the range of $[0.9,1.1] \delta_{\max }$. The COD contours for 
uniform and radially biased distributions are very similar, but the de-adhesion forces differ by about $25 \%$. On the other hand, the de-adhesion forces for the two types of biased distributions in Eqs. (25) and (26) are almost indistinguishable, but the corresponding COD contours are radically different. Consequently, it shows that the clustering of high-affinity integrins in the angular direction does not really change the de-adhesion force noticeably. That is, the more important parameter is $N$, and the less important one is $M$. In fracture mechanics, a similar observation is that a rosette-like crack is no stronger than a circular crack.

Changing the dimensionless number, $c_{11}^{*} \delta_{\max } / \hat{\xi}_{H}^{0} \sigma_{\max } a$, will change the crack bridging behavior. As shown in Fig. 11(a), the de-adhesion forces for $N=4$ are all higher than those for $N=0$. All these curves in Fig. 11(a) approach unity at large $c_{11}^{*} \delta_{\max } / \hat{\xi}_{H}^{0} \sigma_{\max } a$, corresponding to the LSB limit, and these curves should have a slope of $-1 / 2$ at low $c_{11}^{*} \delta_{\max } / \hat{\xi}_{H}^{0} \sigma_{\max } a$ as predicted by the linear elastic fracture mechanics for the SSB limit. It is difficult for simulations with $L_{22} / L_{11}=440$ to reach the SSB limit because of the very fine mesh requirement. The dependence of $F_{\max } / F_{\min }$ on $c_{11}^{*} \delta_{\max } / \hat{\xi}_{H}^{0} \sigma_{\max } a$ shows that the de-adhesion force anisotropy vanishes at the LSB limit. On the other hand, the de-adhesion force anisotropy, $F_{\max } / F_{\min }$, for $N=4$ is smaller than that for $N=0$. As shown in Fig. 11(b), at the LSB limit (i.e., large $c_{11}^{*} \delta_{\max } / \hat{\xi}_{H}^{0} \sigma_{\max } a$ ), the deadhesion process is governed by the interface strength, so the substrate elastic anisotropy plays no role and the ratio of $F_{\max } / F_{\min }$ reaches unity. At the SSB limit (i.e., small $F_{\max } / F_{\min }$ ), the deadhesion force anisotropy reaches a plateau value which is much smaller than the 2D prediction in Chen and Gao (2007). Although $N=4$ shows the same trend, the corresponding curves are lower than those of $N=0$ and also reach much lower plateau values at the SSB limit. These findings suggest that the 2D prediction in Chen and Gao (2007) is unlikely to take place in realistic applications due to contact shape and also biased bond density distributions. In summary, the biased bond density distribution is beneficial for large cell sizes, but has little advantage for small cell sizes. In addition, it should also be noted that from the results in Figs. 4 and 5, the small cells do not tend to form biased bond density distributions.

\section{Summary}


Cell adhesion to the extracellular matrix is accomplished by the clustering of receptorligand bonds on the cell-substrate interface, which is subjected to contractile force by stress

fibers that are connected to the cell cytoskeleton. The interface is modeled by a surface discontinuity and the surrounding by elastic continuum solids. The cohesive interface model specifies a set of constitutive equations relating the traction force and the interfacial separation, where the integrin densities serve as state variables and their evolutions are governed by the conformational conversion of the two types of integrins and the diffusion of the low-affinity integrins. To address the three questions raised in Section 1, we have found the following.

(1) The high affinity and low affinity integrins are always in chemical equilibrium, while the diffusional transport of low affinity ones is driven by a chemical potential gradient that couples concentration and mechanical potential energy. When a cell is subjected to contractile force, the stress gradient from the contact edge to the cell center drives the formation of a higher concentration of receptor-ligand bonds near the contact edge. This phenomenon is more significant at the SSB limit.

(2) We borrow an important conclusion from Bower and Ortiz (1990) that a propagating crack into a decreasing toughness field cannot maintain a straight crack front. The unstable or wavy crack front has a wavelength that is on par with the spatial gradient of the toughness field. To this end, the rosette shape of the adhered cells can be explained.

(3) In contrast to the structural design of bio-adhesive contacts in geckos or generally fibrillar structures, the biased receptor-ligand bond density field, as driven by chemomechanical processes, is found to significantly increase the de-adhesion force. However, the de-adhesion force anisotropy by the substrate elastic anisotropy is reduced quite dramatically.

\section{Acknowledgements}

ZWG acknowledges the financial support from the Natural Science Foundation of China (NSFC 11272140, 10902046), the NSFC Innovative Research Group (11421062), the State Key Project of ITER on Magneto-Constrained Fusion Energy Development Program (2013GB110002B), the State Key Project of Scientific Instrument and Equipment Development (11327802), and the Fundamental Research Funds for the Central Universities (lzujbky-2015176). YFG acknowledges support from the US National Science Foundation (CMMI 1300223). 
We are grateful to Prof. Wei He at the University of Tennessee for fruitful discussions on cell biology, and also to the two reviewers for their thoughtful comments that have significantly improved this work.

\section{Appendix: Finite element formulation}

Solving $\delta \Pi=0$ for Eq. (1) is equivalent to finding the solution of the following variational equation,

$$
\int_{\Omega} \sigma_{i j} \delta \varepsilon_{i j} d \Omega+\int_{S} T_{i} \delta \Delta_{i} d S=\int_{S_{\text {external }}} t_{i}^{*} \delta u_{i} d S
$$

where the second term on the left hand side corresponds to the cohesive interface model. In the implementation in ABAQUS UEL subroutine, provided with the interface separation at $\hat{t}$ and $\hat{t}+\Delta \hat{t}$, we need to update $T_{i}^{\hat{t}+\Delta \hat{t}}, \hat{\xi}_{H}(r, \hat{t}+\Delta \hat{t})$, and $\hat{\xi}_{L}(r, \hat{t}+\Delta \hat{t})$, and also specify the Jacobian, $\partial T_{k} / \partial \Delta_{l}$. Note that time is normalized by $t^{*}=b_{l}^{2} / m k_{B} T$, so that $t=\hat{t} \times t^{*}$.

The finite difference method is employed to solve the diffusion equation in Eq. (10), but the UEL subroutine does not provide access to the neighboring elements. Following Qu et al. (2004, 2006), Gao et al. (2015) and Pu (2015), a common block approach is used to store two

arrays for $\hat{\xi}_{H}\left(r_{k}, \hat{t}\right)$ and $\hat{\xi}_{L}\left(r_{k}, \hat{t}\right)$, where the radial coordinate is discretized into $r_{1}, r_{2}, \ldots, r_{Z}$. Our simulations in Fig. 6 use $Z=101$. The diffusion equation in Eq. (10) now becomes,

$$
\frac{\partial}{\partial \hat{t}}\left\{\hat{\xi}_{L}\left[1+\frac{1}{\beta\left(\Delta_{k}\right)}\right]\right\}=b_{l}^{2} \frac{1}{r} \frac{\partial}{\partial r}\left(r \frac{\partial \hat{\xi}_{L}}{\partial r}\right)=b_{l}^{2}\left[\frac{\partial^{2} \hat{\xi}_{L}}{\partial r^{2}}+\frac{1}{r} \frac{\partial \hat{\xi}_{L}}{\partial r}\right],
$$

and the finite difference form is given by

$$
\begin{aligned}
& \frac{\left[\hat{\xi}_{L}\left(1+\beta^{-1}\right)\right]_{r=r_{j}}^{t=t+\Delta t}-\left[\hat{\xi}_{L}\left(1+\beta^{-1}\right)\right]_{r=r_{j}}^{t=t}}{\Delta \hat{t}}= \\
& b_{l}^{2}\left[\frac{{ }^{t} \hat{\xi}_{L}\left(r_{j+1}\right)-2^{t} \hat{\xi}_{L}\left(r_{j}\right)+{ }^{t} \hat{\xi}_{L}\left(r_{j-1}\right)}{(\Delta r)^{2}}+\frac{1}{r_{j}} \frac{{ }^{t} \hat{\xi}_{L}\left(r_{j+1}\right)-{ }^{t} \hat{\xi}_{L}\left(r_{j-1}\right)}{2 \Delta r}\right]
\end{aligned}
$$

To ensure numerical stability, the Lax condition gives $\Delta \hat{t} \leq \frac{1}{2}\left(\frac{\Delta x}{b_{l}}\right)^{2}$. 


\section{References}

Autumn K, Liang YA, Hsieh, ST, Zesch W, Chan WP, Kenny TW, Fearing R, Full RJ (2000) Adhesive force of a single gecko foot-hair. Nature 405, 681-685.

Bao G, Suo Z (1992) Remarks on crack-bridging concepts. Appl. Mech. Rev. 45, 355-366.

Bower AF (2009) Applied mechanics of solids. CRC Press, Boca Raton, FL, USA.

Bower AF, Ortiz M (1990) Solution of three-dimensional crack problems by a finite perturbation method. J. Mech. Phys. Solids 38, 443-480.

Chen SH, Gao HJ (2007) Bio-inspired mechanics of reversible adhesion: orientation-dependent adhesion strength for non-slipping adhesive contact with transversely isotropic elastic materials. J. Mech. Phys. Solids 55, 1001-1015.

Deshpande VS, McMeeking RM, Evans AG (2006) A bio-chemo-mechanical model for cell contractility. Proc. Natl. Acad. Sci. USA 103, 14015-14020.

Deshpande VS, McMeeking RM, Evans AG (2007) A model for the contractility of the cytoskeleton including the effects of stress-fibre formation and dissociation. Proc. R. Soc. A 463, 787-815.

Deshpande VS, Mrksich M, McMeeking RM, Evans AG (2008) A bio-mechanical model for coupling cell contractility with focal adhesion formation. J. Mech. Phys. Solids 56, 14841510 .

Evans E (2001) Probing the relation between force-lifetime-and chemistry in single molecular bonds. Annu. Rev. Biophys. Biomol. Struct. 30, 105-128.

Freund LB, Lin Y (2004) The role of binder mobility in spontaneous adhesive contact and implications for cell adhesion. J. Mech. Phys. Solids 52, 2455-2472.

Gao HJ (1988) Nearly circular shear mode cracks. Int. J. Solids Struct. 24, 177-193.

Gao HJ (1989) Weight functions for external circular cracks. Int. J. Solids Struct. 25, 107-127.

Gao HJ (2014) Probing mechanical principles of cell-nanomaterial interactions. J. Mech. Phys. Solids 62, 312-339.

Gao HJ, Ji BH, Jäger IL, Arzt E, Fratzl P (2003) Materials become insensitive to flaws at nanoscale: lessons from nature. Proc. Natl. Acad. Sci. USA 100, 5597-600.

Gao HJ, Qian J, Chen B (2011) Probing mechanical principles of focal contacts in cell-matrix adhesion with a coupled stochastic-elastic modeling framework. J. R. Soc. Interface 8, 1217-1232.

Gao HJ, Rice JR (1986) Shear stress intensity factors for a planar crack with slightly curved front. J. Appl. Mech. 53, 774-778.

Gao HJ, Rice JR (1987) Somewhat circular tensile cracks. Int. J. Frac. 33, 155-174.

Gao YF (2010) A Peierls perspective on mechanisms of atomic friction. J. Mech. Phys. Solids 58, 2023-2032.

Gao YF, Bower AF (2004) A simple technique for avoiding convergence problems in finite element simulations of crack nucleation and growth on cohesive interface. Model. Simul. Mater. Sci. Eng. 12, 453-463.

Gao YF, Bower AF (2006) Elastic-plastic contact of a rough surface with Weierstrass profile. Proc. R. Soc. A 462, 319-348.

Gao YF, Larson BC, Lee JH, Nicola L, Tischler JZ, Pharr GM (2015) Lattice rotation patterns and strain gradient effects in face-centered-cubic single crystals under spherical indentation. J. Appl. Mech. 82, 061007.

He SJ, Su YW, Ji BH, Gao HJ (2014) Some basic questions on mechanosensing in cell-substrate interaction. J. Mech. Phys. Solids 70, 116-135. 
Heepe L, Gorb SN (2014) Biologically inspired mushroom-shaped adhesive microstructures, Annu. Rev. Mater. Res. 44, 173-203.

Huang L, Pu C, Fisher RK, Mountain DJH, Gao YF, Liaw PK, Zhang W, He W (2015) A Zrbased bulk metallic glass for future stent applications: materials properties, finite element modeling, and in vitro human vascular cell response. Acta Biomater. 25, 356-368.

Ji BH, Gao HJ (2010) Mechanical principles of biological nanocomposites. Annu. Rev. Mater. Res. 40, 77-100.

Johnson KL (1997) Adhesion and friction between a smooth elastic spherical asperity and a plane surface. Proc. R. Soc. Lond. A 453, 163-179.

Li TL, Lee JH, Gao YF, Pharr GM, Huang M, Tsui TY (2009) Geometric effects on dislocation nucleation in strained electronics. Appl. Phys. Lett. 94, 171905.

Lin Y, Freund LB (2008) Optimum size of a molecular bond cluster in adhesion. Phys. Rev. E 78, 021909.

Liu Y, Gao YF (2015) Non-uniform breaking of molecular bonds, peripheral morphology and releasable adhesion by elastic anisotropy in bio-adhesive contacts. J. R. Soc. Interface 12 , 20141042.

Liu Y, Liu H, Gao YF (2015) Can mushroom-shaped fibers enhance the bio-adhesive performance? J. Mech. Med. Bio. 15, 1550068.

Liu Y, Luo Y, Wang D, Gao YF (2015) Alignment of cellular focal contacts and their shapes by substrate anisotropy. J. Mech. Med. Bio. 15, 1550067.

Maloney JM, Walton EB, Bruce CM, Van Vliet KJ (2008) Influence of finite thickness and stiffness on cellular adhesion-induced deformation of compliant substrata. Phys. Rev. E 78, 041923.

Pathak A, Deshpande VS, McMeeking RM, Evans AG (2008) The simulation of stress fibre and focal adhesion development in cells on patterned substrates. J. R. Soc. Interface 5, 507524.

Pathak A, McMeeking RM, Evans AG, Deshpande VS (2011) An analysis of the cooperative mechano-sensitive feedback between intracellular signaling, focal adhesion development, and stress fiber contractility. J. Appl. Mech. 78, 041001.

$\mathrm{Pu}$ C (2015) Failure simulations at multiple length scales in high temperature structural alloys. $\mathrm{PhD}$ Thesis, University of Tennessee, Knoxville.

Qian J, Wang JZ, Lin Y, Gao HJ (2009) Lifetime and strength of periodic bond clusters between elastic media under inclined loading. Biophys. J. 97, 2438-2445.

Qu S, Huang Y, Jiang H, Liu C, Wu PD, Hwang KC (2004) Fracture analysis in the conventional theory of mechanism-based strain gradient (CMSG) plasticity. Int. J. Fract. 129, 199220.

Qu S, Huang Y, Pharr GM, Hwang KC (2006) The indentation size effect in the spherical indentation of iridium: a study via the conventional theory of mechanism-based strain gradient plasticity. Int. J. Plast. 22, 1265-1286.

Rice JR (1985) First order variations in elastic fields due to variation in location of a planar crack front. J. Appl. Mech. 52, 571-579.

Rice JR (1992) Dislocation nucleation from a crack tip: an analysis based on the Peierls concept. J. Mech. Phys. Solids 40, 239-271

Shao Y, Zhao HP, Feng XQ, Gao HJ (2012) Discontinuous crack-bridging model for fracture toughness analysis of nacre. J. Mech. Phys. Solids 60, 1400-1419. 
Spuskanyuk AV, McMeeking RM, Deshpande VS, Arzt E (2008) The effect of shape on the adhesion of fibrillar surfaces. Acta Biomater. 4, 1669-1676.

Wang JZ, Gao HJ (2010) Size and shape dependent steady-state pull-off force in molecular adhesion between soft elastic materials. Int. J. Fract. 166, 13-19.

Xia SM, Gao YF, Bower AF, Lev LC, Cheng YT (2007) Delamination mechanism maps for a strong elastic coating on an elastic-plastic substrate subjected to contact loading. Int. J. Solids Struct. 44, 3685-3699.

Yao HM, Gao HJ (2006) Mechanics of robust and releasable adhesion in biology: bottom-up designed hierarchical structures of gecko. J. Mech. Phys. Solids 54, 1120-1146.

Zhang WL, Lin Y, Qian J, Chen WQ, Gao HJ (2013) Tuning molecular adhesion via material anisotropy. Adv. Funct. Mater. 23, 4729-4738.

Zhang Z, Yoon J, Suo Z (2006) Method to analyze dislocation injection from sharp features in strained silicon structures. Appl. Phys. Lett. 89, 261912. 


\section{Table Captions}

Table $1 \quad$ Modeling parameters in the boundary value problems in Figs. 2 and 3, where the cohesive interface constitutive law is coupled with the diffusion and conformational conversion of high-affinity and low-affinity integrins. The representative values used in our finite element simulations are compiled from Deshpande et al. (2008), Pathak et al. (2008, 2011), and He et al. (2014).

Table 2 Representative elastic constants of transversely isotropic materials (Chen and Gao, 2007; Liu and Gao, 2015). We intentionally choose a very large ratio of $E_{t} / E_{p}$ in the second row, in order to study the effect of elastic anisotropy on the de-adhesion force anisotropy. The normalization in Figs. 9 and 11 adopt $c_{11}^{*}=23.6$ $\mathrm{kPa}$. 
Table 1

\begin{tabular}{|c|c|c|}
\hline Parameters & Meaning & $\begin{array}{l}\text { Representative values used in finite } \\
\text { element simulations }\end{array}$ \\
\hline$\mu_{H}^{0}-\mu_{L}^{0}$ & $\begin{array}{l}\text { Difference in the reference chemical } \\
\text { potentials of high-affinity and low- } \\
\text { affinity integrins }\end{array}$ & $4.5951 k_{B} T$ \\
\hline$T$ & Absolute temperature & $310 \mathrm{~K}$ \\
\hline$\xi_{0}$ & $\begin{array}{c}\text { Area density of both types of } \\
\text { integrins at } t=0\end{array}$ & $4444 \mu \mathrm{m}^{-2}$ \\
\hline$b_{l}$ & Integrin spacing & $=1 / \sqrt{\xi_{0}}=15 \mathrm{~nm}$ \\
\hline$m$ & Mobility of low-affinity integrins & $10 \mathrm{mg}^{-1} \mathrm{~s}$ \\
\hline$L$ & Characteristic length in Eq. (15) & $20 \mathrm{~nm}$ \\
\hline$k_{b}$ & Receptor-ligand bond stiffness & $0.02 \mathrm{nN} / \mu \mathrm{m}$ \\
\hline$k_{c}$ & $\begin{array}{c}\text { Ideal stiffness in the cohesive } \\
\text { interface model }\end{array}$ & $=\xi_{0} k_{b}=88.88 \mathrm{kPa} / \mu \mathrm{m}$ \\
\hline$\frac{1}{2} k_{b} L^{2}$ & $\begin{array}{c}\text { Characteristic energy of receptor- } \\
\text { ligand bonds }\end{array}$ & $0.935 k_{B} T$ \\
\hline$t^{*}=b_{l}^{2} / m k_{B} T$ & Diffusional time scale & $5.3 \mathrm{~ms}$ \\
\hline$a$ & Cell radius & $10 \mu \mathrm{m}$ \\
\hline$h$ & Cell height & $2 \mu \mathrm{m}$ \\
\hline$E_{c}$ & Elastic stiffness of the cell & $20 \mathrm{kPa}$ \\
\hline
\end{tabular}


Table 2

\begin{tabular}{|c|c|c|c|c|c|c|}
\hline \multirow{2}{*}{$\begin{array}{c}\text { Substrate } \\
\text { Transversely isotropic }\end{array}$} & \multicolumn{6}{|c|}{ Elastic constants } \\
\hline & $c_{11}=23.6$ & $c_{33}=459.2$ & $c_{12}=9.055$ & $c_{13}=12.25$ & $c_{44}=4.0 \mathrm{kPa}$ & $c_{66}=7.27$ \\
\hline \multirow{3}{*}{ material $\left(L_{22} / L_{11}=4.41\right)$} & $\mathrm{kPa}$ & $\mathrm{kPa}$ & $\mathrm{kPa}$ & $\mathrm{kPa}$ & & $\mathrm{kPa}$ \\
\hline & $E_{\mathrm{p}}=20$ & $E_{\mathrm{t}}=450$ & $v_{\mathrm{p}}=0.375$ & $v_{\mathrm{tp}}=0.375$ & $v_{\mathrm{pt}}=0.0167$ & $\mu_{\mathrm{t}}=4 \mathrm{kPa}$ \\
\hline & $\mathrm{kPa}$ & $\mathrm{kPa}$ & & & & \\
\hline Transversely isotropic & $c_{11}=23.27$ & $c_{33}=4.5$ & $c_{12}=8.727$ & $c_{13}=12.0$ & $c_{44}=4.0 \mathrm{kPa}$ & $c_{66}=7.27$ \\
\hline \multirow{4}{*}{$\begin{array}{l}\text { material (fictitious) } \\
\qquad\left(L_{22} / L_{11}=440\right)\end{array}$} & $\mathrm{kPa}$ & $\mathrm{GPa}$ & $\mathrm{kPa}$ & $\mathrm{kPa}$ & & $\mathrm{kPa}$ \\
\hline & & & & & & \\
\hline & $E_{\mathrm{p}}=20$ & $E_{\mathrm{t}}=4.5$ & $v_{\mathrm{p}}=0.375$ & $v_{\mathrm{tp}}=0.375$ & $v_{\mathrm{pt}}=1.67 \times 10^{-6}$ & $\mu_{\mathrm{t}}=4 \mathrm{kPa}$ \\
\hline & $\mathrm{kPa}$ & GPa & & & & \\
\hline
\end{tabular}




\section{Figure Captions}

Figure 1 (a) Focal adhesion of human aortic endothelial cells (HAECs) on a stainless steel substrate, as shown by the immunofluorescent staining image (Huang et al., 2015). (b) Schematic illustration of the clustering of receptor-ligand bonds into discrete focal-adhesion sites at the contact periphery.

Figure 2 Cell adhesion is modeled by an elastic disk on an elastic half-space, subjected to a cohesive constitutive law on the interface. Integrins attached on the cell surface have two different conformational states, denoted as high affinity and low affinity integrins. The integrin and bond densities provide state variables for the cohesive interface model, where a bilinear model is illustrated as an example.

Figure 3 Essential ideas for the formation of discrete focal-adhesion sites on the contact periphery. (a) Initial state. (b) From the Eshelby point of view, cell contraction is equivalent to the application of peripheral tractions, or viewed as Mode-II crack at the contact edge. (c) Stress-induced evolution of high-affinity and low-affinity integrins leads to a higher (or lower) density of high-affinity integrins at the contact edge (or center), thus leading to a radial gradient in the cohesive energy density. (d) For a crack propagating into a decreasing toughness field, the crack font will become unstable and wavy. (e) Final equilibrium state.

Figure 4 (a) An axisymmetric model of cell adhesion subjected to contractile tractions. The interface is modeled by a linear relationship with ideal stiffness being $k_{c}$. (b) Shear stress distribution for perfectly bonded interface or large $k_{c} \hat{\xi}_{H}$, corresponding to the small-scale-bridging (SSB) behavior. (c) Shear stress distribution and interface separation for a low value of $k_{c} \hat{\xi}_{H}$, showing the largescale-bridging (LSB) behavior.

Figure 5 Finite element simulation results of shear stress distributions along the interface in Fig. 4(a). The two curves with triangles and inverted triangles denoted with linear springs and fixed integrin densities demonstrate the SSB-LSB transition, while the curve with variable integrin densities illustrates the coupling between stress and integrin density fields. These two figures are of the same data, but plotted in linear and logarithmic scales, respectively, for the vertical axis. 
Figure 6 The evolution of integrin density fields, $\hat{\xi}_{L}=\xi_{L} / \xi_{0}$ and $\hat{\xi}_{H}=\xi_{H} / \xi_{0}$, for lowaffinity and high-affinity ones respectively, with respect to the contractile traction. The normalized applied strain rate is $\left|\dot{\sigma}_{\text {appl }} t^{*} / E\right|=10^{-2}$. Other modeling parameters can be found in Table 1.

Figure 7 Schematic illustration of the perturbation analysis of a straight Mode-II crack front propagating into a decreasing toughness field, based on Bower and Ortiz (1990).

Figure 8 The distributed bond density fields as modeled in Eqs. (25) and (26). (a) Radial distribution only with $N=4$. (b) Both radial and angular distribution with $N=4$ and $M=6$.

Figure 9 (a) De-adhesion simulation of a circular contact on a transversely isotropic material where $p$ direction lies in the plane of transverse isotropy and $t$ is the out-of-plane direction. (b) Representative load-displacement curves with the change of the bond density fields, subjected to a shear load in the $p$ direction. The radial distribution of the bond density field substantially increases the deadhesion force, while an additional angular distribution contributes negligibly to the de-adhesion force. Modeling parameters include $L_{22} / L_{11}=440$, $\hat{\xi}_{H}^{0} \sigma_{\max } / c_{11}^{*}=0.08$ and $\delta_{\max } / a=0.0025$.

Figure 10 Contour plots of the crack opening displacement (COD), i.e., "Field-1", for the de-adhesion simulations in Fig. 9. (a) \& (b) Uniform bond density field (Liu and Gao, 2015). (c) Nonuniform bond density field with radial distribution only. (d) Nonuniform bond density field with both radial and angular distributions. (a), (c), and (d) correspond to the peak loads of the three curves in Fig. 9(b). The crack fronts can be delineated by these COD contours since the COD value is bounded in a narrow range near $\delta_{\max }$.

Figure 11 De-adhesion forces with respect to the SSB-to-LSB transition. (a) Both maximum and minimum forces approach the SSB (or Griffith) limit for small $c_{11}^{*} \delta_{\max } / \hat{\xi}_{H}^{0} \sigma_{\max } a$, and approaches the LSB (or uniform stress) limit for large $c_{11}^{*} \delta_{\max } / \hat{\xi}_{H}^{0} \sigma_{\max } a$. The nonuniform bond density distributions make it more 
difficult to separate the bio-adhesive contacts. (b) The anisotropy in the deadhesion force with respect to the loading direction, $F_{\max } / F_{\min }$. The nonuniform bond density distributions reduce the de-adhesion force anisotropy. 


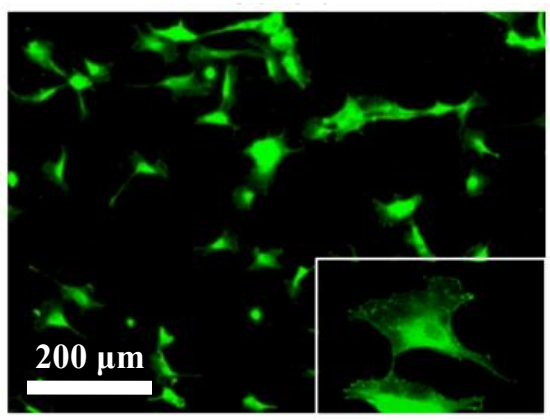

(b)

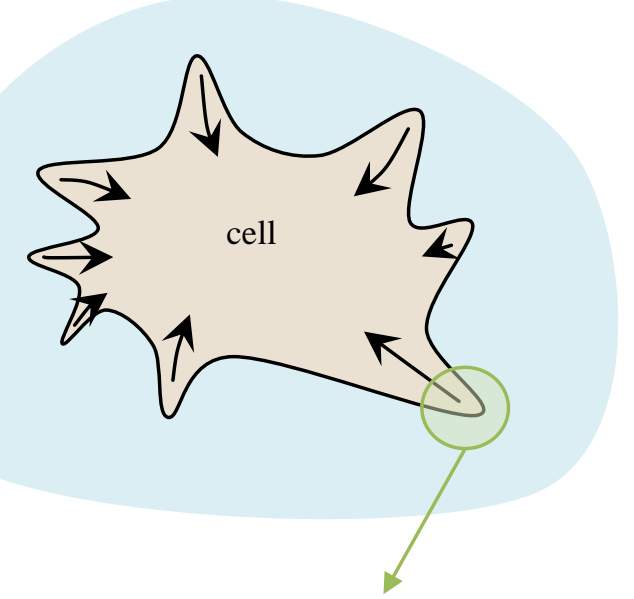

High density of receptorligand bonds

Figure 1 


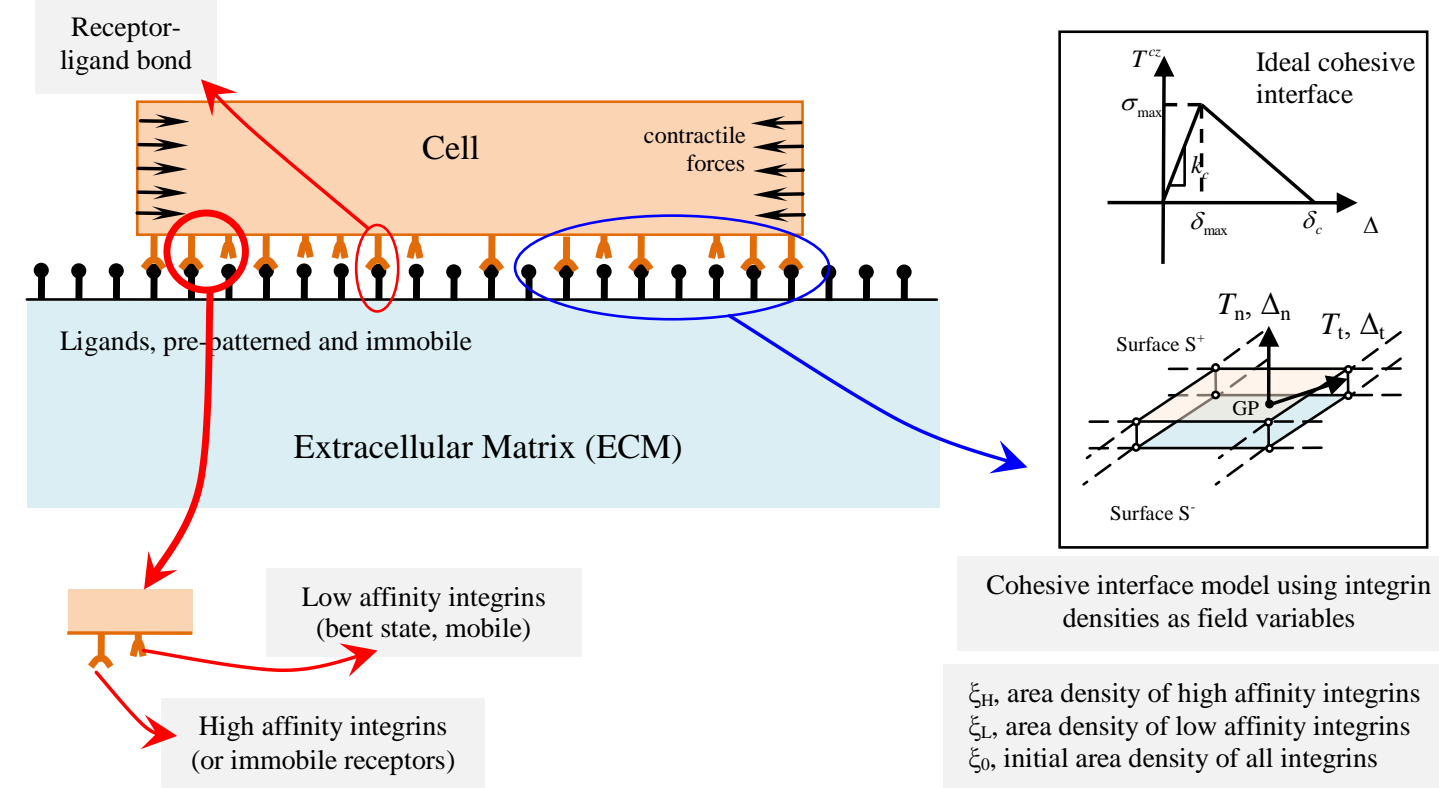

Figure 2 
(a)

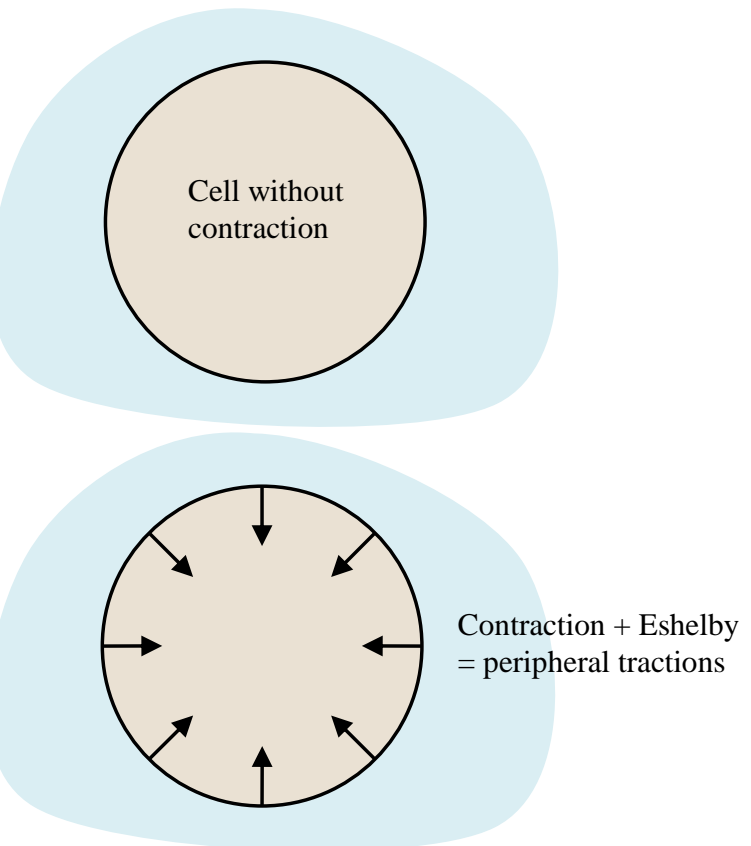

(c)

Integrin evolution ? a radial gradient in toughness (or cohesive energy)

(d)

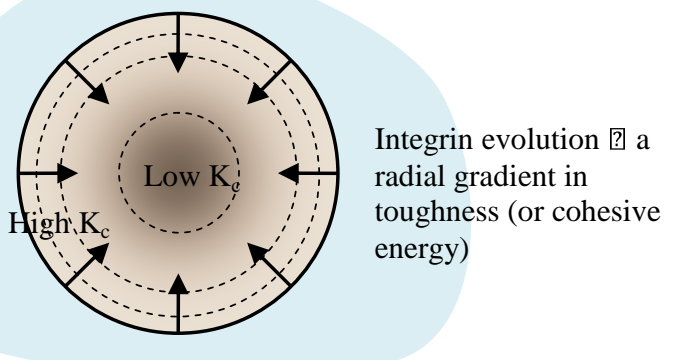

(e)
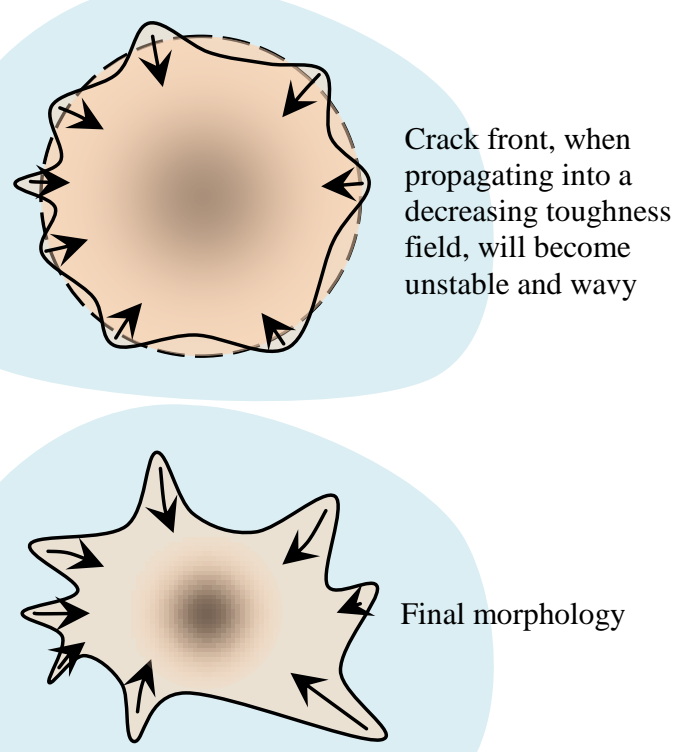

Figure 3 


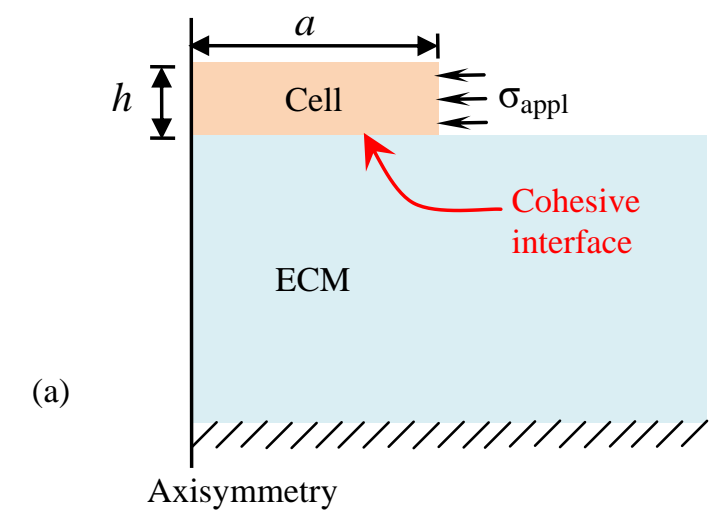

(b)
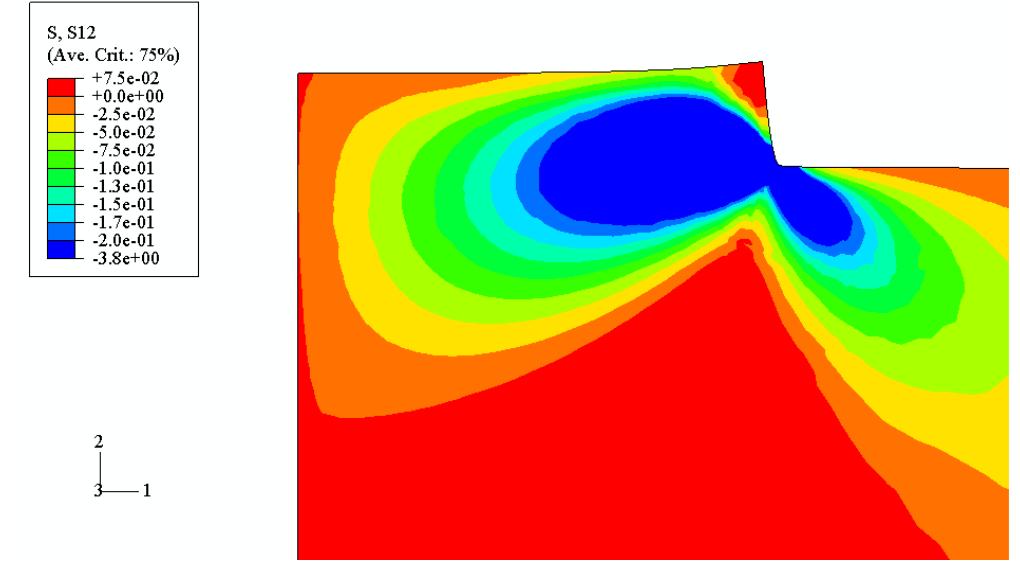

(c)
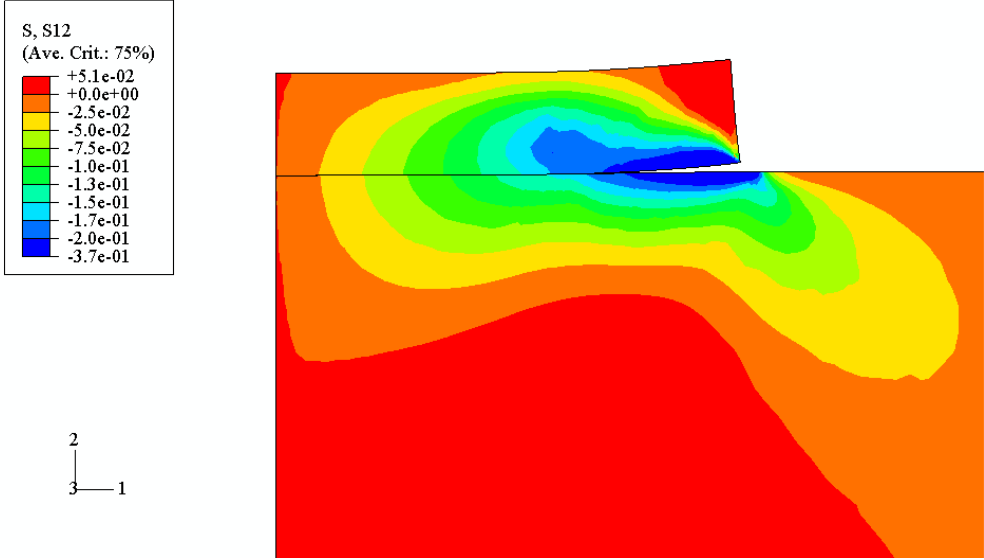

Figure 4 


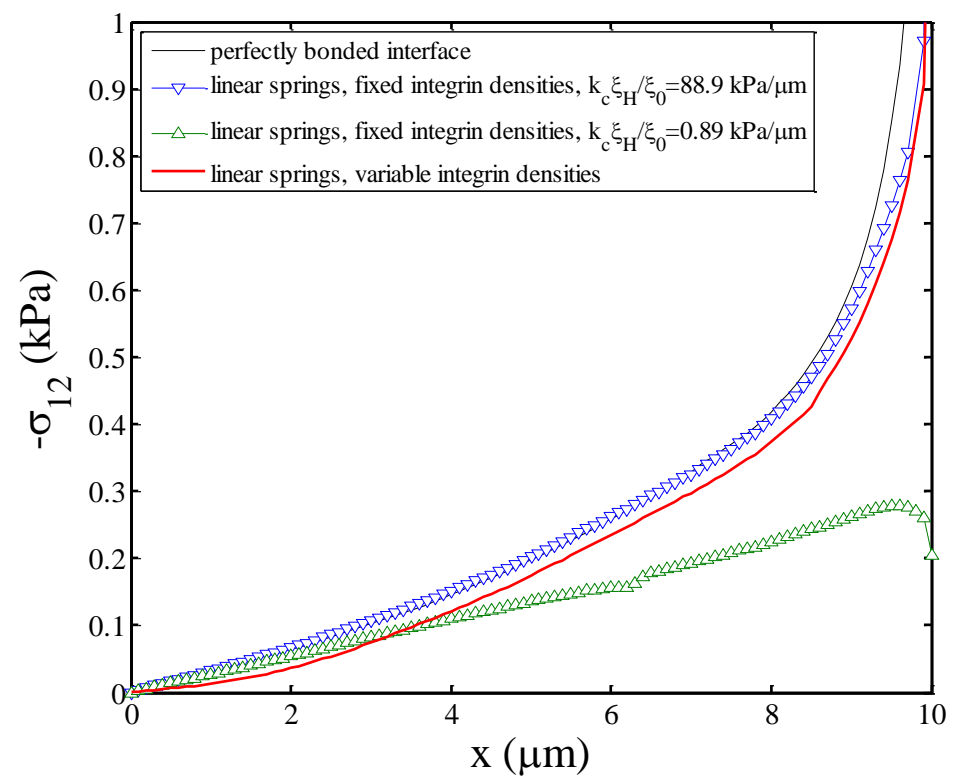

(a)

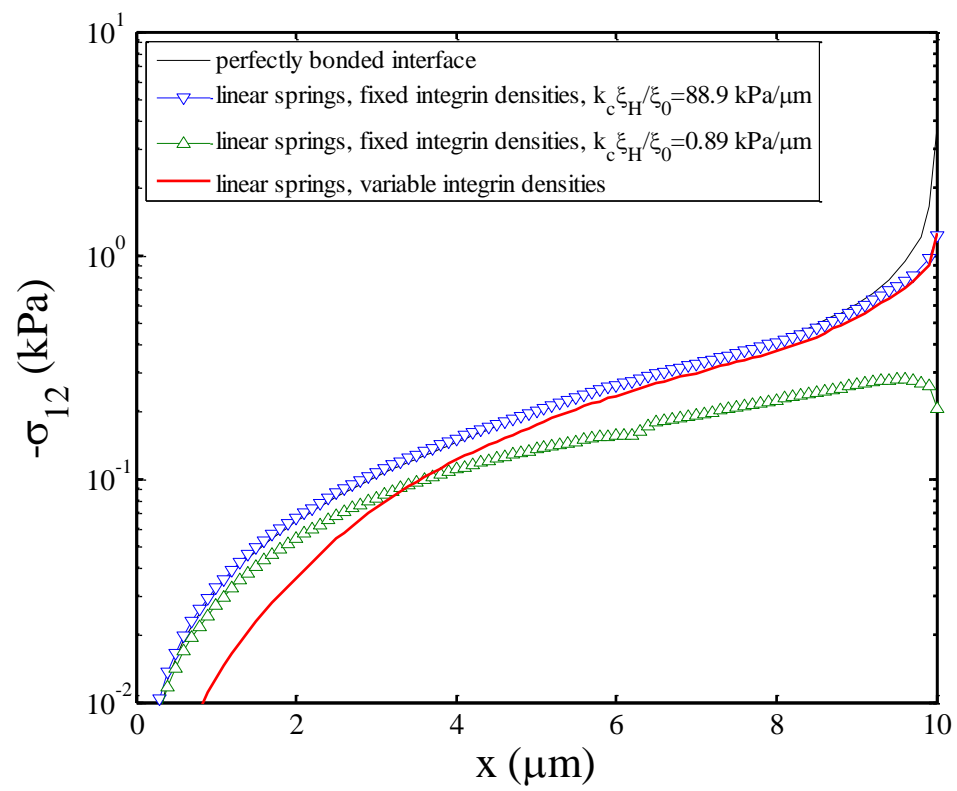

(b)

Figure 5 


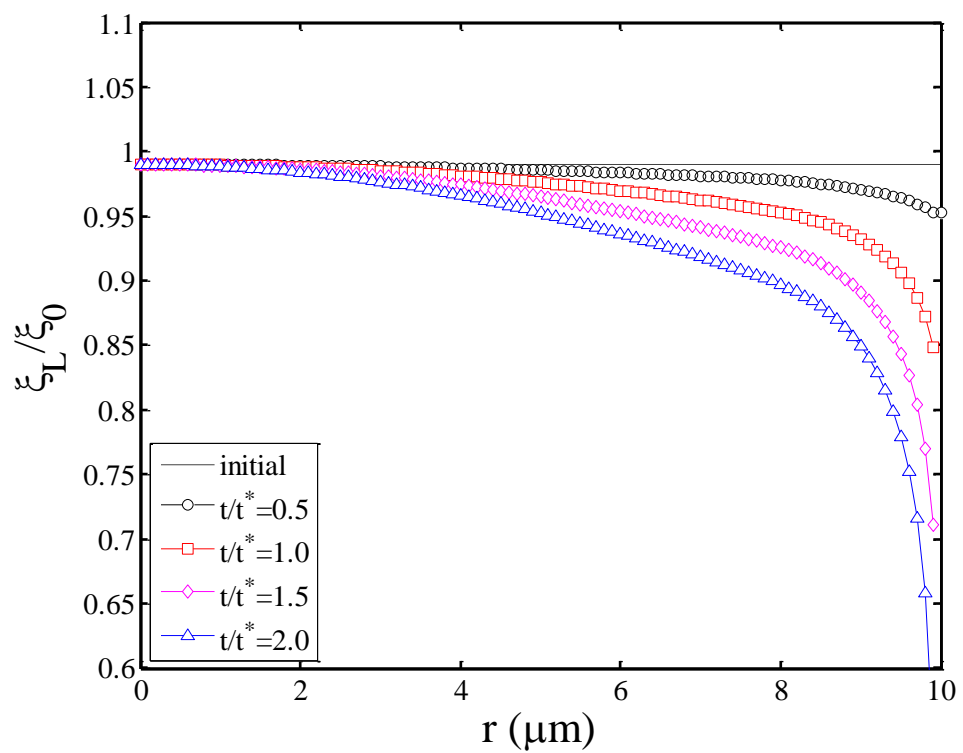

(a)

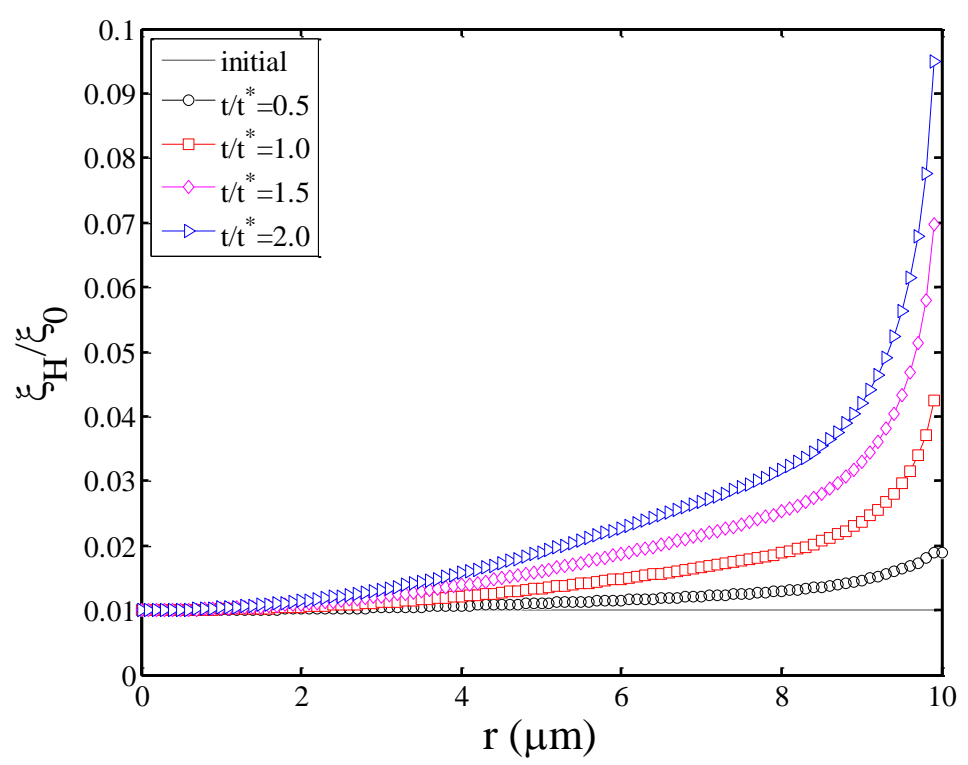

(b)

Figure 6 


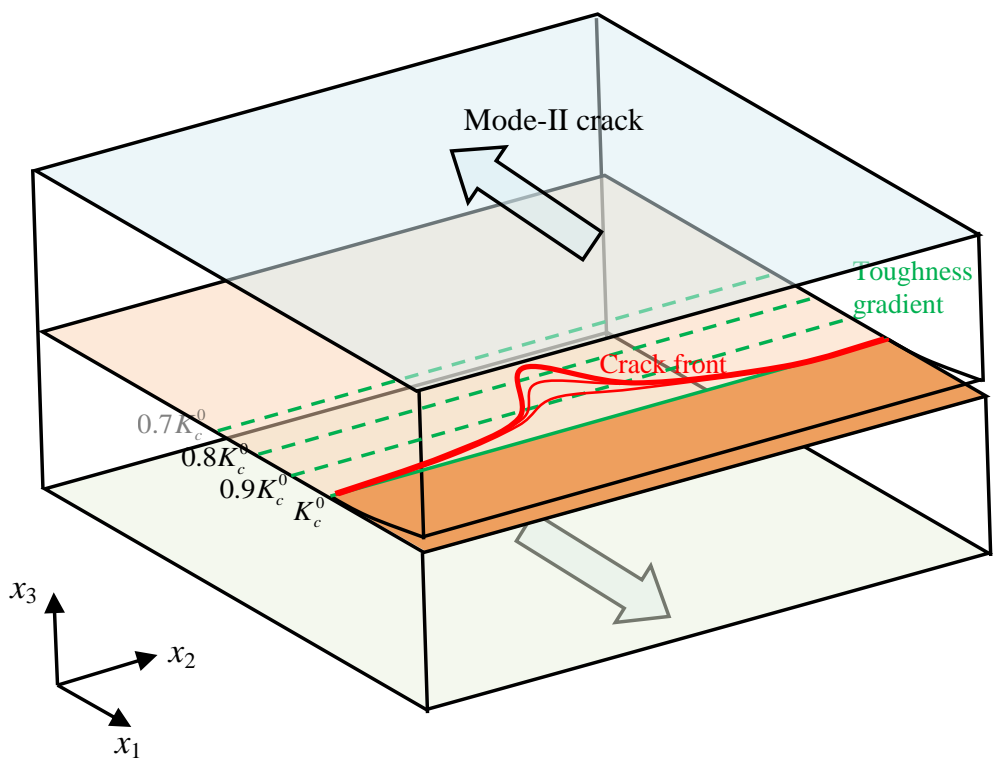

Figure 7 


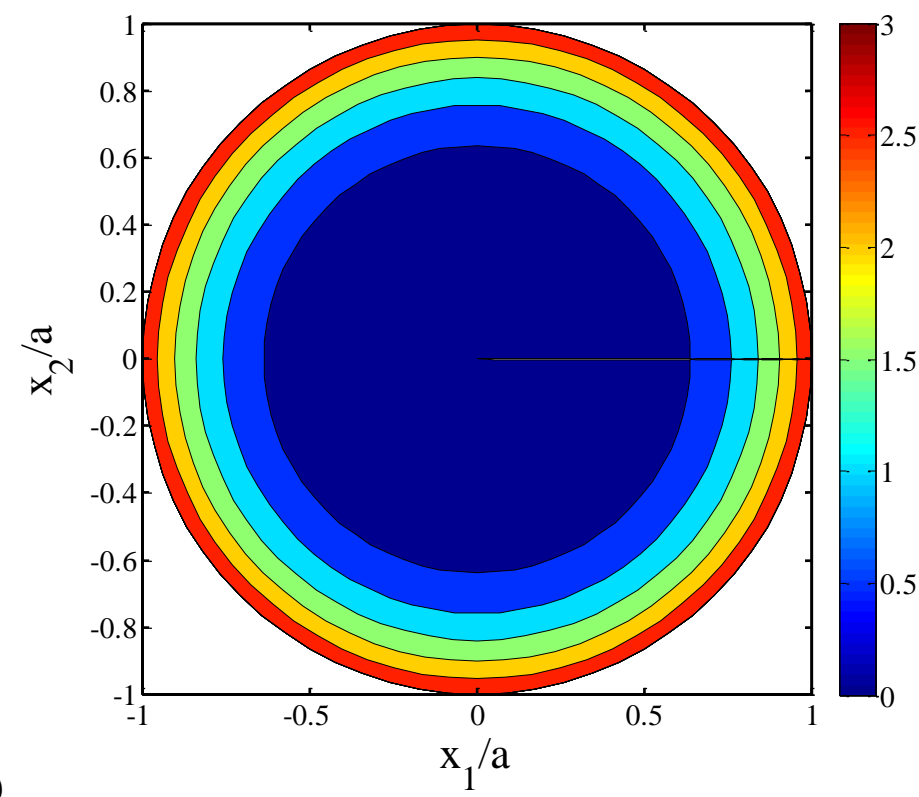

(a)

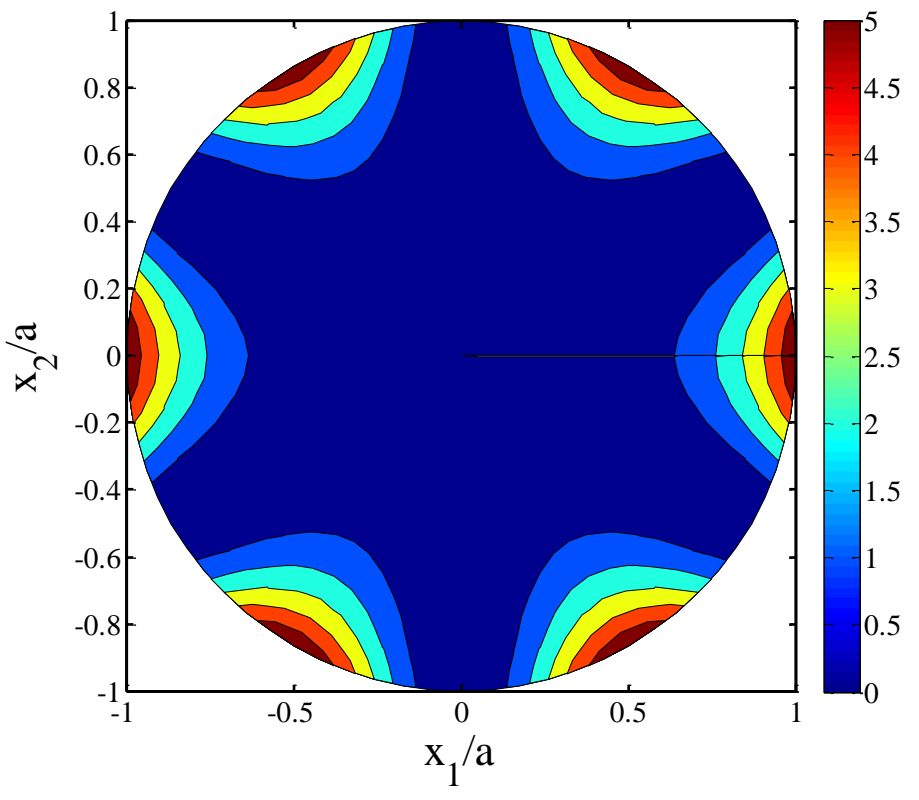

Figure 8 
(a)
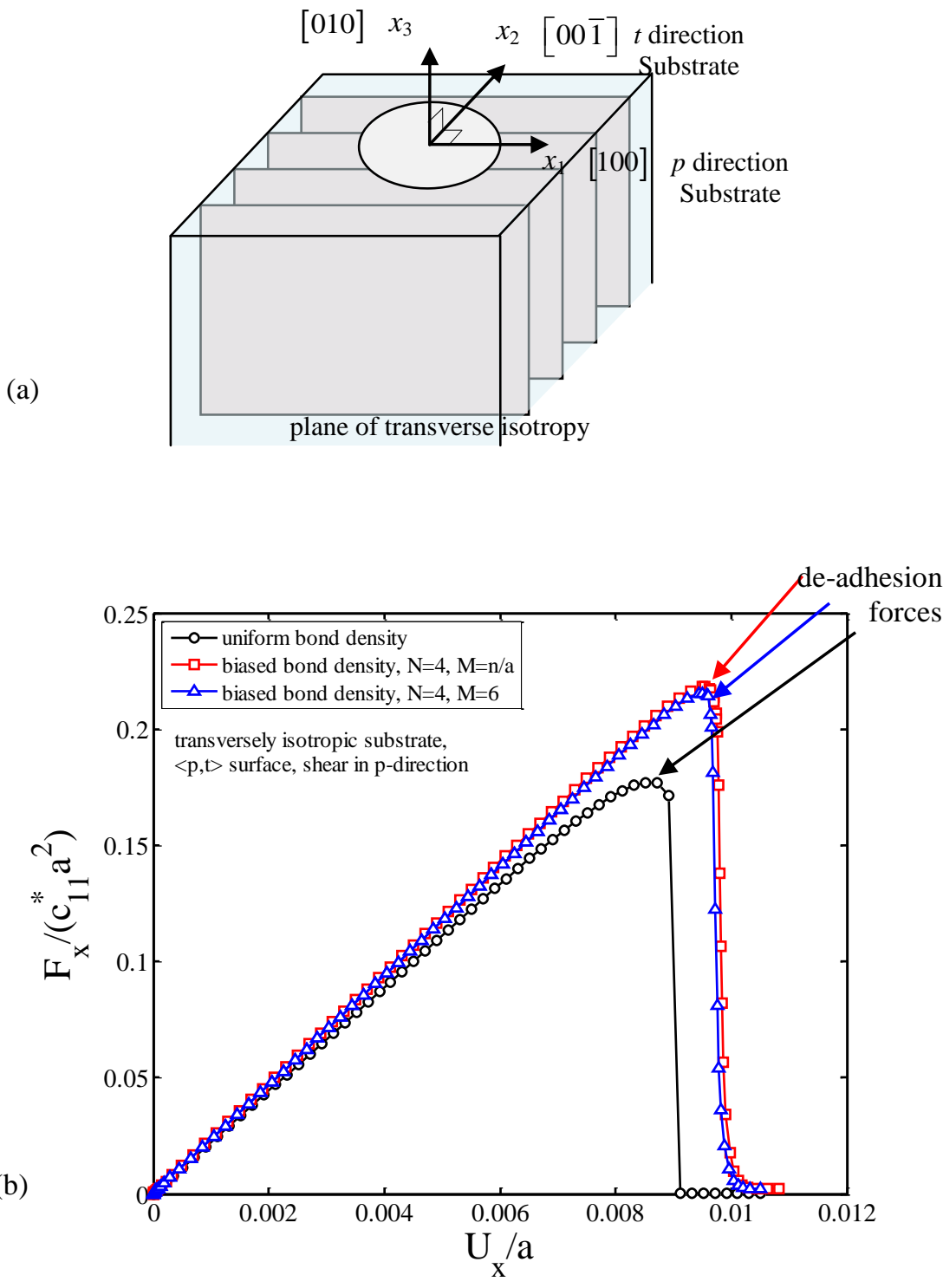

Figure 9 

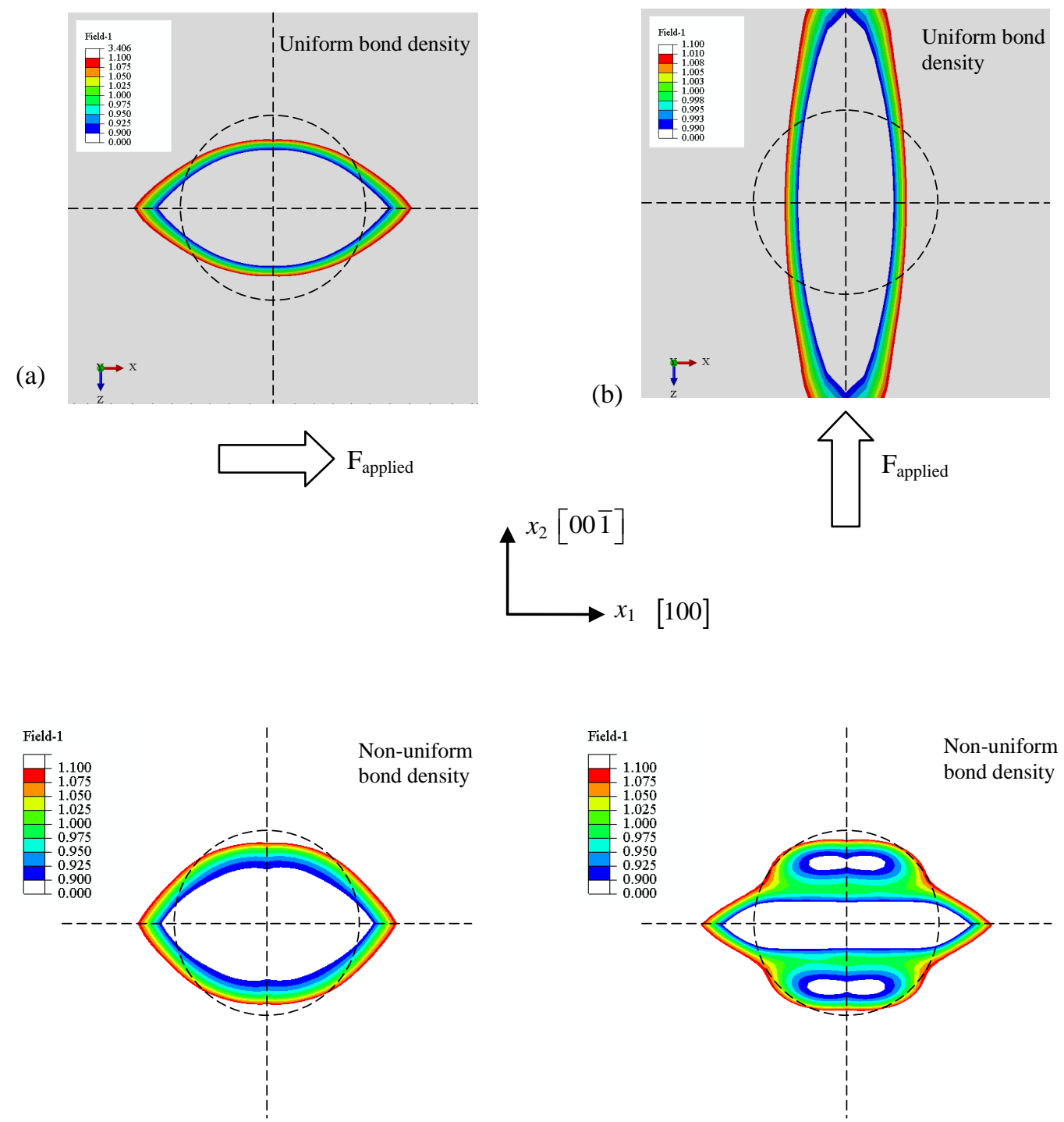

(c)

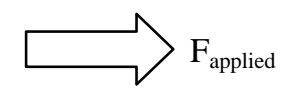

(d)

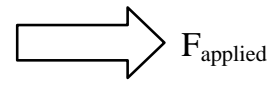

Figure 10 
(a)
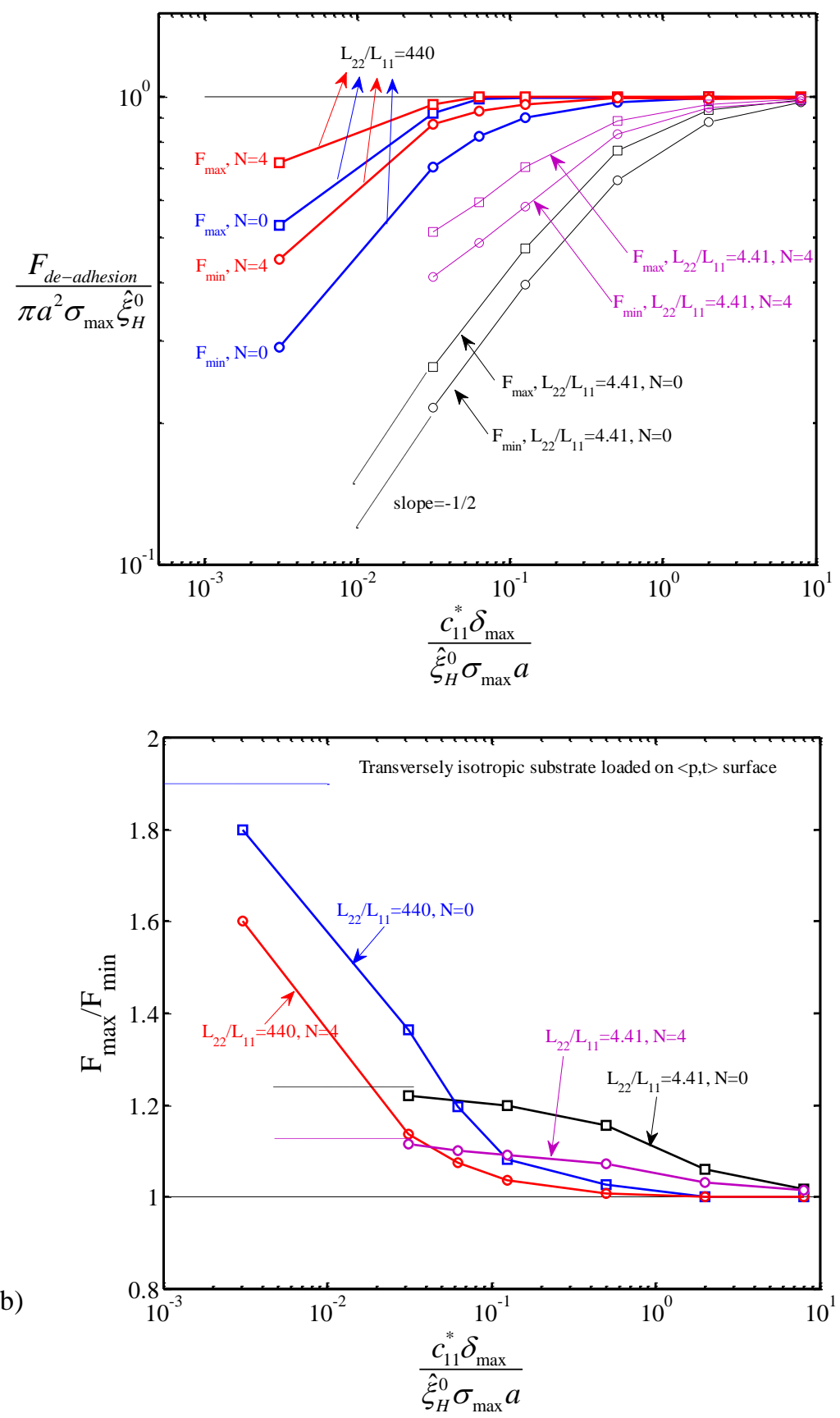

Figure 11 